\title{
Numerische Simulationen des Ausbreitungsverhaltens von Wärmeträgerfluid-Inhaltsstoffen aus Erdwärmesonden in Trinkwassereinzugsgebieten
}

\author{
Janine Struß ${ }^{1}$ D $\cdot$ Dirk Schäfer $^{1} \cdot$ Andreas Dahmke $^{1} \cdot$ Ralf Köber $^{1}$ \\ Eingegangen: 26. Juni 2019 / Überarbeitet: 20. Januar 2020 / Online publiziert: 19. Juni 2020 \\ (c) Der/die Autor(en) 2020
}

\section{Zusammenfassung}

Aufgrund deutlich erhöhter Wärmebedarfsdichten urbaner Räume besteht in besonderem Maße hier die Möglichkeit und Erfordernis, durch eine nachhaltige Wärmeversorgung und saisonale Wärmespeicherung im geologischen Untergrund einen wesentlichen Beitrag zu den national und international gesetzten Klimaschutzzielen zu liefern. Eine effiziente Möglichkeit zur Wärmegewinnung und -speicherung bieten hierfür Erdwärmesonden, die jedoch aus Gründen des vorbeugenden Grundwasserschutzes in Bereichen der Trinkwassernutzung heutzutage meist nicht oder nur beschränkt genehmigt werden. Numerische Simulationen einer Erdwärmesondenleckage für Randbedingungen eines zur Trinkwassergewinnung genutzten norddeutschen Aquifers auf Grundlage von aufgearbeiteten stoffspezifischen Abbauratenkonstanten zeigen, dass Grenzwerte für die meisten handelsüblichen Wärmeträgerfluid-Inhaltsstoffe bereits bei einem Abstand von nur $100 \mathrm{~m}$ zwischen Erdwärmesonde und Trinkwasserentnahme aufgrund starker Verdünnung und mikrobiellen Abbaus mit einem Faktor $>10$ unterschritten werden. Vor dem Hintergrund dieser Ergebnisse und angesichts der Zielsetzung einer Reduktion fossiler Primärenergiequellen um 80-95\% bis 2050, erscheinen pauschale Abstandsvorgaben (von zurzeit $>1000 \mathrm{~m}$ ) daher zumindest hinsichtlich der Trinkwassergefährdung durch Erdwärmesondenleckagen diskussionswürdig.

\section{Numerical simulations of a borehole heat exchanger leakage in drinking water catchment areas}

\begin{abstract}
Given the necessity to provide a substantial contribution to the climate protection goals borehole heat exchangers (BHE) and thermal energy storages (BTES) provide an efficient way to produce and store heat, especially in urban areas, but are for reasons of preventive groundwater protection usually rarely approved in areas of drinking water production. Numerical simulations of a BHE leakage in an aquifer used for drinking water production show that even at a distance of only $100 \mathrm{~m}$ between the BHE and the drinking water production well concentrations for most components used in commercially available heat transfer fluids do not exceed the limit values due to microbial degradation and dilution. Considering these results and in view of the ambition to reduce fossil primary energy sources by $80-95 \%$ by 2050 general distance requirements (currently $>1000 \mathrm{~m}$ ) therefore seem worthy of discussion, at least with regard to the drinking water pollution caused by leakages of BHE.
\end{abstract}

Keywords Borehole heat exchanger - Heat transfer fluids · Additives · Groundwater - Risk assessment

\author{
Janine Struß \\ janine.struss@ifg.uni-kiel.de \\ Dirk Schäfer \\ dirk.schaefer@ifg.uni-kiel.de \\ Andreas Dahmke \\ andreas.dahmke@ifg.uni-kiel.de
}

Ralf Köber

ralf.koeber@ifg.uni-kiel.de

1 Institut für Geowissenschaften,

Christian-Albrechts-Universität zu Kiel,

Ludewig-Meyn-Str. 10, 24118 Kiel, Deutschland 


\section{Einleitung}

Im Zuge der Energiewende wurde mit dem Erneuerbare-Energien-Gesetz beschlossen, eine nachhaltige Entwicklung der Wärme- und Kälteversorgung zu ermöglichen und die Weiterentwicklung der Technologien zur Nutzung erneuerbarer Energien zu fördern. Während der Anteil erneuerbarer Energien im Stromsektor von 2014 bis 2018 von 27,4 auf 37,8\% gesteigert werden konnte, liegt der Anteil erneuerbarer Energien im Wärmesektor 2018 bei 14,4\% (BMWi 2019). Dabei ist die Umstellung auf erneuerbare Energien im Wärmesektor von zentraler Bedeutung, um den Primärenergiebedarf im Gebäudebestand bis 2050 um $80 \% \mathrm{zu}$ senken. Besonders die fluktuierenden erneuerbaren Energiequellen und die strombasierte Versorgung von Gebäuden mit Niedertemperaturwärme für Raumheizung und Warmwasser werden in der zukünftigen Energieversorgung eine wichtige Rolle spielen. Daher ist der Ausbau von Wärmenetzen in Kombination mit großen Wärmespeichern zum Ausgleich von Energiedefiziten essenziell, um die Zielsetzung der Bundesregierung einer Reduktion der Treibhausgas-Emissionen um 80 bis $95 \%$ bis 2050 gegenüber 1990 zu erreichen. Die Deckung des Raumwärmebedarfs macht mehr als 50\% aller Endenergieverbrauch-Sektoren aus (FVEE 2015) und gerade im urbanen Raum, wo der Wärmebedarf am höchsten ist, bietet der geologische Untergrund die potenziell größten Wärmespeicherkapazitäten, sowohl zum Kühlen als auch zum Heizen von Gebäuden, sodass vor allem Mega- und Großstädte in Zukunft eine Schlüsselrolle bei der Umsetzung von Klimaschutzmaßnahmen einnehmen werden.

Die Erschließung des Untergrundes zur Wärmespeicherung oder für die oberflächennahe geothermische Heizung und Kühlung von Gebäuden kann mit Erdwärmesonden mit einer hohen Leistungsdichte erzielt werden (Koenigsdorff und Reuß 2006). Sie sind der häufigste Anlagentyp unter den Erdwärmepumpensystemen und können in Deutschland $\mathrm{CO}_{2}$-Einsparungen von $30 \%$ und mehr gegenüber herkömmlichen Heizanlagen wie Öl und Gas bewirken (Saner et al. 2010). Sie werden in vertikalen Bohrungen meist mit Tiefen bis $100 \mathrm{~m}$ installiert und bestehen in der Regel aus Doppel-U-Rohr-Sonden mit einem Innendurchmesser von $26 \mathrm{~mm}$. In den Sondenrohren zirkuliert ein Wärmeträgerfluid, welches meist aus Wasser und einem Frostschutzmittel besteht. Am häufigsten wird Monoethylenglykol als Frostschutzmittel verwendet, gefolgt von Propylenglykol (Rumohr 2009). Andere Wärmeträgerfluide wie $\mathrm{CO}_{2}$, Ethanol und Calciumchlorid sind hingegen nur wenig vertreten (Rumohr 2009). Dem Wärmeträgerfluid werden meistens zu verschiedenen Zwecken Additive beigefügt, welche als Korrosionsinhibitoren, Biozide, Farb- und Duftstoffe oder Stabilisatoren dienen (Klotzbücher et al. 2007). MengenmäBig am stärksten vertretene Additive sind Korrosionsinhibi- toren wie Benzo- und Tolyltriazole, sowie Ethylhexansäure, Karbonsäuren (Sebacin- und Benzoesäure) und Amine (Ilieva et al. 2012).

Es handelt sich bei den Sonden zwar um geschlossene Systeme, ein Austritt der Fluide bei Leckagen kann jedoch nicht völlig ausgeschlossen werden. Leckagen können durch Undichtigkeiten verursacht werden, die entweder durch Fehler bei der Installation, Materialverschleiß, Korrosion oder Schäden durch äußere Einwirkung entstehen (Bonte et al. 2011). Gemeldete Fälle defekter Erdwärmesonden, bei denen ein Druckabfall im System und somit ein möglicher Austritt des Wärmeträgerfluids detektiert wurde, liegen schätzungsweise $<1 \%$ der installierten Erdwärmesondenanlagen (Ad-Hoc-Arbeitsgruppe Geologie 2011). Weitere Aspekte, denen hier jedoch nicht nachgegangen wird, sind Auswirkungen, die durch das Schaffen oder Plombieren von Wegsamkeiten im Untergrund, und somit einer Änderung der hydraulischen Verhältnisse entstehen, da es dadurch u.a. zum Austreten von artesisch gespanntem Grundwasser, Versiegen von Quellen und zur Änderung der chemischen Zusammensetzung des Grundwassers kommen kann (Bonte et al. 2011; Butscher et al. 2011; Grimm et al. 2014; LAWA 2011; Köber et al. 2015; Lüders et al. 2016, 2020). Eine Abschätzung der Wahrscheinlichkeit von Schadensfällen bei Erdwärmebohrungen in Baden-Württemberg ergab eine Eintrittswahrscheinlichkeit für einen Schadensfall von unter $0,002 \%$ pro Jahr, wobei die Ursache in $90 \%$ der Fälle eine unvollständige, undichte Hinterfüllung der Erdwärmesonde bei kritischem Stockwerksbau oder gespannten Grundwasser war (Grimm et al. 2014).

Derzeit ist nach DVGW (2010) in Trinkwassereinzugsgebieten die Nutzung von Erdwärme in den Schutzzonen I, II und III/IIIA aus Vorsorgegründen zu unterlassen. Da in der Regel die Gefährdung des zu fördernden Grundwassers mit zunehmender Verweilzeit durch Dispersions-, Abbau- und Sorptionsprozesse abnimmt, können die Nutzungsbeschränkungen nach DVGW (2010) in der weiteren Schutzzone abgestuft werden, sodass im Einzelfall Ausnahmen in der Schutzzone IIIB möglich sind, wenn ausschließlich Wärmeträgerfluide verwendet werden, die nicht wassergefährdend sind. Da es bisher noch kein spezielles bundesweites Gesetz für die oberflächennahe Geothermie gibt, werden die Richtlinien aus den Wassergesetzen der Länder, Bundesberg- und Wasserhaushaltsgesetz abgeleitet, sodass die Wasserbehörden gemäß den ländereigenen Richtlinien unterschiedlichen Vorgaben folgen. In einigen Bundesländern ist dabei der Abstand der Erdwärmesonde von Trinkwasserförderbrunnen und in anderen die Lage in den Wasserschutzzonen maßgeblich für deren Zulässigkeit, z.T. werden auch beide Kriterien herangezogen, sodass kein allgemein gültiger Standard besteht. Innerhalb der Wasserschutzzonen I und II von Wasserschutzgebieten 
und in einem Umkreis von $100 \mathrm{~m}$ um die Brunnen der öffentlichen Trinkwasserversorgung ist die Einrichtung von Erdwärmesonden generell unzulässig. In Schleswig-Holstein und Hamburg kann in einer Entfernung von $100 \mathrm{~m}$ bis $1000 \mathrm{~m}$ von Brunnen der öffentlichen Wasserversorgung die Einrichtung außerhalb des Nutzhorizontes gegebenenfalls zulässig sein, wenn der zur Erdwärmegewinnung genutzte Grundwasserleiter durch schützende Deckschichten vom zur Trinkwassergewinnung genutzten Grundwasserleiter getrennt ist. Bei der Einrichtung im zur Trinkwassergewinnung genutzten Grundwasserleiter muss hingegen eine Entfernung von mehr als $1000 \mathrm{~m}$ im Anstrom zur Fassung eingehalten werden, während beispielsweise in Niedersachsen, Sachsen-Anhalt und Mecklenburg-Vorpommern die Einrichtung ab der Schutzzone IIIB zulässig sein kann, welche mindestens $1000 \mathrm{~m}$ und zumindest 50 Tage Fließzeit von der Fassungsanlage entfernt sein sollte, in der Regel jedoch erst ab einer Distanz von $2000 \mathrm{~m}$ beginnt (DVGW 2010). Die Einrichtung von Erdwärmesonden in Trinkwassereinzugsgebieten ist zudem mit erhöhten Schutzanforderungen verbunden. So ist beispielsweise in Hamburg die Einrichtung von Erdwärmesonden in einer Entfernung von $1000 \mathrm{~m}$ bis $2000 \mathrm{~m}$ von Brunnen der öffentlichen Wasserversorgung im Nutzhorizont nur zulässig, wenn nicht wassergefährdende Stoffe, wie z. B. Wasser oder ein Wasser-Glykol-Gemisch mit einem Glykolanteil unter $3 \%$, eingesetzt werden.

Bisher erfolgten zwar Einschätzungen zum Gefährdungspotenzial von Wärmeträgerfluiden hinsichtlich ihrer Einsatzhäufigkeit und ihres Umweltverhaltens, jedoch keine Konkretisierung in Bezug auf die räumliche und zeitliche Ausbreitung. Um den Bedarf an quantitativen Kenntnissen über das Ausbreitungsverhalten von Wärmeträgerfluid-Inhaltsstoffen zu decken und somit eventuell eine optimierte und größere Nutzung regenerativer Energien zu ermöglichen, wurde in dieser Studie das Ausbreitungsverhalten von Wärmeträgerfluid-Inhaltsstoffen im Grundwasser im Einflussbereich einer Trinkwasserentnahme untersucht. Das primäre Ziel lag dabei auf der Quantifizierung der Auswirkungen der berücksichtigten Prozesse auf die Konzentrationsverläufe von Wärmeträgerfluid-Inhaltsstoffen in den Brunnen einer Trinkwasserentnahme zur Bewertung der Relevanz von Erdwärmesondenleckagen. Hierbei wird auf Grundlage von aufgearbeiteten stoffspezifischen Abbauratenkonstanten anhand eines vereinfachten Beispiels das Abbauverhalten von Ethylenglykol, Benzotriazol, Tolyltriazol, 2-Ethylhexansäure, Benzoesäure und Sebacinsäure unter verschiedenen Redoxbedingungen, sowie vergleichsweise auch zum konservativen Transport ermittelt.

\section{Methodik}

Zur Berücksichtigung des Abbauverhaltens potenziell grundwassergefährdender Wärmeträgerfluid-Inhaltsstoffe innerhalb der Simulationen des Ausbreitungsverhaltens dieser Substanzen, war es erforderlich, im ersten größeren Teil dieser Studie stoffspezifische Abbauratenkonstanten aus vorliegenden Untersuchungen zusammenzustellen und aufzuarbeiten.

Nach bisherigen Einschätzungen zum Gefährdungspotential von Wärmeträgerfluid-Inhaltsstoffen wurden diese als potenziell grundwassergefährdend eingeschätzt, wenn sie eine hohe Mobilität im Grundwasser aufweisen und eine eingeschränkte mikrobielle Abbaubarkeit und/oder eine toxische Wirkung gegenüber aquatischen Organismen zeigen (Ilieva et al. 2012). Häufig verwendete Additive, auf die diese Eigenschaften zutreffen, sind z.B. 1-H-Benzotriazol, Tolyltriazol, 2-Ethylhexansäure, Sebacinsäure und Benzoesäure (Tab. 1). So führen die zugefügten Additive im Vergleich zu den reinen Frostschutzkomponenten meist zu einer deutlich höheren Toxizität gegenüber aquatischen Organismen als von den Hauptkomponenten abgeleitet werden kann (Tab. 1). Die Mobilität der Additive, abgeleitet von der Wasserlöslichkeit und dem Oktanol-Wasser-Verteilungskoeffizienten $\left(\log \mathrm{K}_{\mathrm{ow}}\right.$ ), ist meist moderat (Tolyltriazol, 2-Ethylhexansäure, Benzoesäure, Sebacinsäure) bis gut (Benzotriazol), Ethylenglykol ist zudem sehr gut wasserlöslich (Tab. 1).

Ein Vergleich der Abbauratenkonstanten dieser Wärmeträgerfluid-Inhaltsstoffe aus verschiedenen Studien deutet größtenteils auf eine typische Abhängigkeit des Abbaus vom Redoxmilieu hin, bei der die Abbauraten von aeroben über nitratreduzierende $\mathrm{zu}$ fermentativen, eisen(III)reduzierenden und sulfatreduzierenden Bedingungen hin abnehmen (Abb. 1; Tab. 2). Die in Wärmeträgerfluiden gebräuchlichsten Frostschutzmittel Ethylen- und Propylenglykol sind im Gegensatz zu den Triazolen aerob und anaerob gut abbaubar (Carnegie und Ramsay 2009; Gooden 1998; Ilieva et al. 2014; Klecka et al. 1993; McGahey und Bouwer 1992; Schoenberg et al. 2001; Schmidt et al. 2012). Die Mehrzahl der Studien zur Abbaubarkeit von Triazolen weisen auf eine prinzipielle aerobe Abbaubarkeit hin (Heesel 2010; Herzog et al. 2013; Liu et al. 2011, 2013; Mishra et al. 2011; Spahr et al. 2013; Weiss et al. 2006). In einigen Studien konnte jedoch nur ein geringes bzw. kein aerobes Abbaupotenzial festgestellt werden (Hem et al. 2000; Jia et al. 2006; Rollinson und Callely 1986; Schmidt et al. 2012). Unter anaeroben Bedingungen wurde in den meisten Studien nur eine geringe (Schmidt et al. 2012) oder keine Abbaubarkeit nachgewiesen (Hem et al. 2000; Herzog et al. 2013; Hollingsworth et al. 2005). Nur in Untersuchungen von Liu et al. $(2011,2013)$ konnte ein anaerobes Abbaupotenzial von Triazolen gezeigt werden. 
Tab. 1 Mobilität und Toxizität von Wärmeträgerfluid-Inhaltsstoffen. Dargestellt sind Angaben zur Ökotoxizität durch den Geringfügigkeitsschwellenwert (GFS) und die predicted no effect concentration (PNEC), sowie die mittlere effektive Konzentration (EC50) und mittlere lethale Konzentration (LC50) drei trophischer Stufen. Angaben zur Mobilität können der Wasserlöslichkeit und dem n-Oktanol-Wasser-Verteilungskoeffizienten $\left(\mathrm{K}_{\text {ow }}\right)$ entnommen werden

Table 1 Mobility and Toxicity of Heat Transfer Fluid Ingredients. Ecotoxicity data are presented by the threshold values for insignificance (GFS) and the predicted no effect concentration (PNEC), as well as the mean effective concentration (EC50) and mean lethal concentration (LC50) of three trophic levels. Information on mobility can be obtained from water solubility and the n-octanol-water partition coefficient (K $\mathrm{K}_{\mathrm{ow}}$ )

\begin{tabular}{|c|c|c|c|c|c|c|c|}
\hline & \multicolumn{2}{|c|}{ Richtwerte } & \multicolumn{3}{|l|}{ Toxizität } & \multicolumn{2}{|l|}{ Mobilität } \\
\hline & $\begin{array}{l}\text { GFS } \\
{[\mathrm{mg} / \mathrm{l}]}\end{array}$ & $\begin{array}{l}\mathrm{PNEC} \\
{[\mathrm{mg} / \mathrm{l}]}\end{array}$ & $\begin{array}{l}\text { EC50 Daphnia } \\
{[\mathrm{mg} / \mathrm{l}]}\end{array}$ & $\begin{array}{l}\text { LC50 Fisch } \\
{[\mathrm{mg} / \mathrm{l}]}\end{array}$ & $\begin{array}{l}\text { EC50 Bakterien } \\
{[\mathrm{mg} / \mathrm{l}]}\end{array}$ & $\begin{array}{l}\text { Wasserlöslichkeit } \\
{[\mathrm{g} / \mathrm{l}]}\end{array}$ & $\log K_{\text {ow }}$ \\
\hline Ethylenglykol & - & $10^{\mathrm{m}}$ & $48.582-60.407^{f}$ & $\begin{array}{l}>10.000^{\mathrm{f}}, \\
72.860^{\mathrm{a}}\end{array}$ & - & $1000^{\mathrm{m}}$ & $-1,4^{\mathrm{m}}$ \\
\hline Benzotriazol & $0,04^{\mathrm{g}}$ & $0,04^{\mathrm{g}}$ & $102^{\mathrm{j}}$ & $65^{\mathrm{j}}$ & $41^{\mathrm{j}}$ & $19,8^{\mathrm{k}}$ & $1,4^{\mathrm{k}}$ \\
\hline Tolyltriazol & $0,04^{\mathrm{g}}$ & $0,075^{\mathrm{g}}$ & $108^{j}$ & $38^{j}$ & $7^{\mathrm{j}}$ & $4^{a}-5^{c}$ & $1,1^{\mathrm{b}}$ \\
\hline $\begin{array}{l}\text { 2-Ethylhexan- } \\
\text { säure }\end{array}$ & - & $0,36^{\mathrm{i}}$ & $85^{\mathrm{i}}, 120^{\mathrm{d}}$ & $180^{\mathrm{i}}, 150^{\mathrm{d}}$ & $112^{\mathrm{i}}, 43^{\mathrm{d}}$ & $1,4^{\mathrm{i}}$ & $2,6^{\mathrm{k}}-2,7^{\mathrm{i}}$ \\
\hline Sebacinsäure & - & $0,018^{\mathrm{a}}$ & $613^{e}$ & $1056^{\mathrm{e}}$ & - & $1^{\mathrm{h}}$ & $2,2^{\mathrm{h}}$ \\
\hline Benzoesäure & - & $0,33^{1}$ & $500^{f}$ & $180^{\mathrm{f}}$ & $9^{f}$ & $2,9^{\mathrm{f}, 1}-3,5^{\mathrm{a}}$ & $1,9^{f, l}$ \\
\hline
\end{tabular}

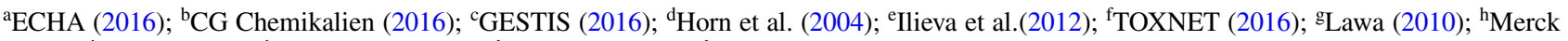
(2013); ${ }^{\mathrm{i} O X E A}$ (2015); ${ }^{\mathrm{j} P i l l a r d ~ e t ~ a l . ~(2001) ; ~}{ }^{\mathrm{P}}$ hysProb (2016); ${ }^{\mathrm{R}}$ Roth (2015a); ${ }^{\mathrm{m}}$ Roth (2015b)

2-Ethylhexansäure bzw. -hexanoat konnte in Abbauversuchen sowohl unter aeroben (EPA 2016; Schmidt et al. 2012) als auch anaeroben Bedingungen (Chua et al. 2001; Schmidt et al. 2012) meist vollständig abgebaut werden. Über den mikrobiellen Abbau von Sebacinsäure bzw. Sebacat ist bisher nur wenig bekannt. In zwei Studien konnte der Abbau von Sebacat unter aeroben Bedingungen dokumentiert werden (Chapman und Duggleby 1967; Siotto et al. 2012). Der Abbau unter anaeroben Bedingungen wurde in einer Studie von Matthies und Schink (1993) bestätigt $\left(1,7 \mathrm{~d}^{-1}\right)$. Der aerobe und anaerobe Abbau von Benzoesäure bzw. Benzoat konnte in einer Vielzahl von Studien gezeigt werden (z. B. Gibson und Sulfita 1986; Kazumi et al. 1995; TOXNET 2016; Williams und Evans 1975).

Die Modellsimulationen erfolgten mit der Open-SourceSoftware OpenGeoSys (Kolditz et al. 2012; Kolditz und Bauer 2004), welche entwickelt wurde, um gekoppelte thermische, hydraulische, mechanische und chemische Prozesse im porösen Medium numerisch zu simulieren. Sie basiert auf der Finite-Elemente-Methode, die zur numerischen Lösung partieller Differentialgleichungen verwendet wird.

Die Modellsimulationen sind angelehnt an einen realen Standort eines Wasserwerkes in Schleswig-Holstein (LANU 2000) und bilden nach einer Parameterstudie von Dethlefsen et al. (2017) häufig auftretende Randbedingungen eines oberflächennahen norddeutschen Aquifers ab. Dazu wurde ein gut durchlässiger Lockergesteins-Aquifer mit einem Durchlässigkeitsbeiwert $\left(\mathrm{k}_{\mathrm{f}}\right)$ von $5 \cdot 10^{-4} \mathrm{~m} / \mathrm{s}$, einem effektiven Porenanteil $\left(\mathrm{n}_{\text {peff }}\right)$ von $20 \%$ aus Busch und Luckner (1974) zitiert nach Garling und Dittrich (1979) und einem natürlichen hydraulischen Gradienten von $2 \cdot 10^{-3}$ (Druckhöhenunterschied $=10 \mathrm{~m}$, Fließstrecke $=5000 \mathrm{~m}$ ) gewählt, wodurch sich eine natürliche Abstandsgeschwindig- keit von $5 \cdot 10^{-6} \mathrm{~m} / \mathrm{s}(0,4 \mathrm{~m} / \mathrm{d})$ ergab. Die Dispersivitäten $\alpha_{\mathrm{L}}$ und $\alpha_{\mathrm{T}}$ wurden aus Gelhar et al. (1985) zitiert nach Rausch et al. (2002) longitudinal mit $10 \mathrm{~m}$ und transversal mit $1 \mathrm{~m}$ abgeschätzt. Das Modell bildet einen gespannten oberflächennahen Grundwasserleiter mit einer Mächtigkeit von $20 \mathrm{~m} \mathrm{ab}$, der ohne Wechselwirkungen mit weiteren Formationen im Hangenden oder Liegenden simuliert wurde. Da ein Worst-Case-Szenario betrachtet wurde, wurde eine Grundwasserneubildung, die lokal zu einer zusätzlichen Konzentrationsverringerung führen würde, nicht berücksichtigt. Über drei vollverfilterte Brunnen, die in Abständen von $80 \mathrm{~m}$ zueinander transversal zur Fließrichtung gesetzt wurden (Abb. 2), erfolgte eine Trinkwasserentnahme mit einer Gesamtentnahmerate von $1.000 .000 \mathrm{~m}^{3} / \mathrm{a}$, welche eine mittlere Entnahmerate für Wasserwerke in SchleswigHolstein darstellt (LLUR 1998). Eine Doppel-U-RohrSonde (32 $\mathrm{mm}$ Sonde mit $26 \mathrm{~mm}$ Innendurchmesser) durchteuft den im Modell betrachteten Aquifer (Abb. 2). Die Erdwärmesondenleckage wurde anhand eines Worst-CaseSzenarios simuliert, bei dem die gesamte Menge des Wärmträgerfluides (2121 für eine $100 \mathrm{~m}$ lange Sonde) mit einer Leckagerate von $200 \mathrm{ml} / \mathrm{h}$ durch eine punktuelle Undichtigkeit in der Sonde, wie z.B. durch Korrosion, $5 \mathrm{~m}$ unter der oberen Schichtgrenze des Aquifers austritt, und sich die Sonde innerhalb von 44 Tagen komplett entleert.

Die Diskretisierung des Modellgebietes erfolgte mit einem dreidimensionalen Finite-Elemente-Gitter über ein $5000 \mathrm{~m} \times 5000 \mathrm{~m}$ großes Gebiet mit einer konstanten Mächtigkeit von $20 \mathrm{~m}$. Für das Gitter wurde im Großteil des Modellgebietes eine Zellgröße von $20 \mathrm{~m} \times 20 \mathrm{~m} \times 5 \mathrm{~m}$ verwendet und graduell auf $5 \mathrm{~m} \times 5 \mathrm{~m} \times 5 \mathrm{~m}$ zwischen Erdwärmesonde und Brunnen verfeinert. 


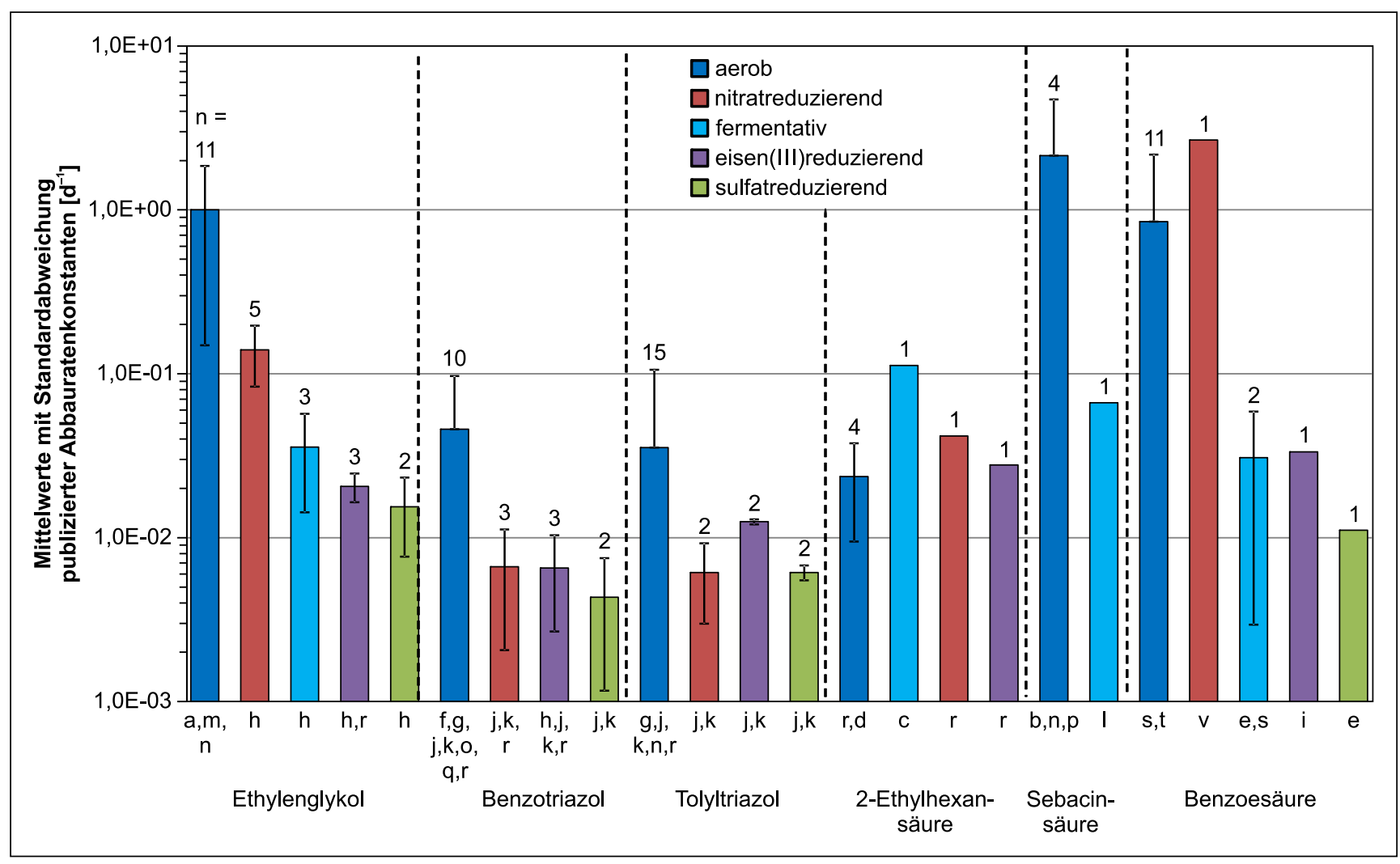

Abb. 1 Abbauratenkonstanten des mikrobiellen Abbaus von Wärmeträgerfluid-Inhaltsstoffen bei verschiedenen Redoxbedingungen. ${ }^{\mathrm{a} C a r n e g i e}$ und Ramsay (2009), ${ }^{\mathrm{b}}$ Chapman und Duggleby (1967), ${ }^{\mathrm{c}}$ Chua et al. (2001), ${ }^{\mathrm{d}} \mathrm{EPA}(2016),{ }^{\mathrm{e}}$ Gibson und Sulfita (1986), ${ }^{\mathrm{f}} \mathrm{Heesel}(2010),{ }^{\mathrm{g}} \mathrm{Herzog}$ et al.

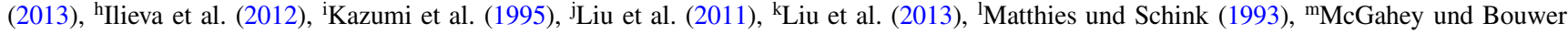
(1992), ${ }^{\mathrm{n}}$ Merck (2013), ${ }^{\mathrm{o}}$ Mishra et al. (2011), ${ }^{\mathrm{P} S}$ Siotto et al. (2012), ${ }^{\mathrm{q}}$ Spahr et al. (2013), ${ }^{\mathrm{r}}$ Schmidt et al. (2012), ${ }^{\mathrm{s}}$ TOXNET (2016), ${ }^{\mathrm{t} W a r d}$ (1985), ${ }^{\text {u} W e i s s ~ e t ~ a l . ~(2006), ~}{ }^{\mathrm{v}}$ Williams und Evans (1975)

Fig. 1 Degradation rate constants of the microbial degradation of heat transfer fluid constituents under various redox conditions. ${ }^{\mathrm{a} C a r n e g i e}$ and Ramsay (2009), ${ }^{\mathrm{b}}$ Chapman and Duggleby (1967), ${ }^{\mathrm{c}}$ Chua et al. (2001), ${ }^{\mathrm{d}} \mathrm{EPA}$ (2016), ${ }^{\mathrm{e}}$ Gibson and Sulfita (1986), ${ }^{\mathrm{f}} \mathrm{Heesel}(2010),{ }^{\mathrm{g}} \mathrm{Herzog}$ et al. (2013), hIlieva et al. (2012), ${ }^{\mathrm{i} K a z u m i}$ et al. (1995), ${ }^{\mathrm{j} L i u}$ et al. (2011), ${ }^{\mathrm{k}}$ Liu et al. (2013), ${ }^{\mathrm{l} M a t t h i e s}$ and Schink (1993), ${ }^{\mathrm{m} M c G a h e y}$ and Bouwer (1992), ${ }^{\mathrm{n}}$ Merck (2013), ${ }^{\circ}$ Mishra et al. (2011), ${ }^{\mathrm{P} S i o t t o}$ et al. (2012), ${ }^{\mathrm{q}} \mathrm{Spahr}$ et al. (2013), ${ }^{\mathrm{r}} \mathrm{S}$ Chmidt et al. (2012), ${ }^{\mathrm{s}}$ TOXNET (2016), ${ }^{\mathrm{t}} \mathrm{Ward}(1985)$, "Weiss et al. (2006), 'Williams and Evans (1975)

Die Ausbreitung des Wärmeträgerfluids im Untergrund wurde für sechs häufig verwendete und zum Teil als potenziell problematisch für Grundwassersysteme angesehene Inhaltsstoffe unter Berücksichtigung von Dispersion und Diffusion auf der Transportstrecke, Verdünnung im Brunnen und mikrobiellem Abbau der Stoffe untersucht. Für die Simulationen wurde das Frostschutzmittel Ethylenglykol, sowie die Korrosionsinhibitoren Benzotriazol, Tolyltriazol, 2-Ethylhexansäure, Sebacinsäure und Benzoesäure ausgewählt und sowohl unter konservativem als auch reaktivem Transport für unterschiedliche Redoxbedingungen simuliert, welcher die Abbaubarkeit der Inhaltsstoffe in jeweils einzelnen Szenarien durch Fermentation oder Nitratreduktion bzw. Sulfatreduktion berücksichtigt (Tab. 2). Die Eingabe-Konzentrationen der Inhaltsstoffe wurden nach maximalen Endkonzentrationen in fertig gemischten Wärmeträgerfluiden für Erdwärmesonden nach Schmidt et al. (2012) und Ilieva et al. (2012) gewählt (Tab. 3).
Der fermentative Abbau wurde über eine Abbaukinetik erster Ordnung in das Modell integriert, bei der die Konzentration $C[\mathrm{mg} / \mathrm{l}]$ der Inhaltsstoffe in Abhängigkeit von einer Ratenkonstante erster Ordnung $k_{l}\left[\mathrm{~d}^{-1}\right]$ über die Zeit $t$ [s] abnimmt:

$\frac{d C_{\text {Stoff }}}{d t}=-k_{1 \text { ferm }} \cdot C_{\text {Stoff }}$

Der Abbauterm für Nitrat- und Sulfatreduktion wurde durch einen Monodterm erweitert, sodass der Abbau an die Konzentration des jeweiligen Elektronenakzeptors gekoppelt ist und nicht stattfindet, wenn diese nicht in ausreichender Konzentration vorhanden sind. Die Halbsättigungskonzentration $K_{\mathrm{H}}$ ist für die untersuchten Stoffe aus der Literatur nicht gegeben. Aus der Abwasserreinigung ist jedoch bekannt, dass bei der Denitrifikation bei $>1-2 \mathrm{mg} / \mathrm{l}$ Nitrat die Reaktionsgeschwindigkeit von der Nitratkonzentration unabhängig ist (Kunz 2013). Die Halbsättigungskonzentration $K_{\mathrm{H}}\left(10^{-3} \mathrm{mg} / \mathrm{l}\right)$ wurde daher so gewählt, dass diese sehr 
Tab. 2 Abbauratenkonstanten erster Ordnung des mikrobiellen Abbaus von Wärmeträgerfluid-Inhaltsstoffen unter aeroben ( $\mathrm{k}_{1-\mathrm{aarob}}$ ), fermentierenden ( $\mathrm{k}_{1 \text {-ferm}}$ ), nitratreduzierenden ( $\left.\mathrm{k}_{1-\mathrm{NO} 3}\right)$, sulfatreduzierenden ( $\left.\mathrm{k}_{1-\mathrm{SO}}\right)$ und Eisen(III)-reduzierenden ( $\mathrm{k}_{1}$-Fe(III) $)$ Bedingungen mit Mittelwert $\overline{\mathrm{x}}$ und Standardabweichung $\sigma$

Table 2 First order degradation rate constants of the microbial degradation of heat transfer fluid constituents under aerobic ( $\left.\mathrm{k}_{1 \text {-aerob}}\right)$, fermenting $\left(\mathrm{k}_{1 \text {-ferm}}\right)$, nitrate-reducing ( $\left.\mathrm{k}_{1-\mathrm{NO} 3}\right)$, sulphate-reducing ( $\left.\mathrm{k}_{1-\mathrm{SO} 4}\right)$ and iron(III)-reducing $\left(\mathrm{k}_{1-\mathrm{Fe}(\mathrm{IIII})}\right)$ conditions with mean $\overline{\mathrm{x}}$ and standard deviation $\sigma$

\begin{tabular}{|c|c|c|c|c|c|c|}
\hline & & $\mathrm{k}_{1 \text {-aerob }\left[\mathrm{d}^{-1}\right]}$ & $\mathrm{k}_{1 \text {-ferm }\left[\mathrm{d}^{-1}\right]}$ & $\mathrm{k}_{1-\mathrm{NO} 3}\left[\mathrm{~d}^{-1}\right]$ & $\mathrm{k}_{1-\mathrm{SO} 4}\left[\mathrm{~d}^{-1}\right]$ & $\mathrm{k}_{1-\mathrm{Fe}(\mathrm{III})}\left[\mathrm{d}^{-1}\right]$ \\
\hline \multirow[t]{4}{*}{ Ethylenglykol ${ }^{\mathrm{a}, \mathrm{h}, \mathrm{m}, \mathrm{n}, \mathrm{r}}$} & Min & $5,0 \cdot 10^{-2}$ & $2,0 \cdot 10^{-2}$ & $8,0 \cdot 10^{-2}$ & $9,9 \cdot 10^{-3}$ & $1,8 \cdot 10^{-2}$ \\
\hline & $\operatorname{Max}$ & 2,9 & $6,0 \cdot 10^{-2}$ & $2,0 \cdot 10^{-1}$ & $2,1 \cdot 10^{-2}$ & $2,5 \cdot 10^{-2}$ \\
\hline & $\overline{\mathrm{x}}$ & 1,0 & $3,6 \cdot 10^{-2}$ & $1,4 \cdot 10^{-1}$ & $1,6 \cdot 10^{-2}$ & $2,1 \cdot 10^{-2}$ \\
\hline & $\sigma$ & $8,1 \cdot 10^{-1}$ & $1,7 \cdot 10^{-2}$ & $5,1 \cdot 10^{-2}$ & $5,6 \cdot 10^{-3}$ & $3,3 \cdot 10^{-3}$ \\
\hline \multirow[t]{4}{*}{ Benzotriazol $^{\mathrm{f}, \mathrm{g}, \mathrm{j}, \mathrm{h}, \mathrm{k}, \mathrm{o}, \mathrm{q}, \mathrm{r}}$} & Min & $4,4 \cdot 10^{-3}$ & - & $2,1 \cdot 10^{-3}$ & $1,6 \cdot 10^{-3}$ & $5,6 \cdot 10^{-4}$ \\
\hline & Max & $1,4 \cdot 10^{-1}$ & - & $1,1 \cdot 10^{-2}$ & $6,0 \cdot 10^{-3}$ & $1,1 \cdot 10^{-2}$ \\
\hline & $\overline{\mathrm{x}}$ & $4,1 \cdot 10^{-2}$ & - & $5,0 \cdot 10^{-3}$ & $3,8 \cdot 10^{-3}$ & $4,1 \cdot 10^{-2}$ \\
\hline & $\sigma$ & $4,9 \cdot 10^{-2}$ & - & $2,7 \cdot 10^{-3}$ & $2,6 \cdot 10^{-3}$ & $3,1 \cdot 10^{-3}$ \\
\hline \multirow[t]{4}{*}{ Tolyltriazol ${ }^{\mathrm{g}, \mathrm{j}, \mathrm{k}, \mathrm{n}, \mathrm{r}}$} & Min & $4,4 \cdot 10^{-4}$ & - & $3,9 \cdot 10^{-3}$ & $5,7 \cdot 10^{-3}$ & $1,2 \cdot 10^{-2}$ \\
\hline & $\operatorname{Max}$ & $2,4 \cdot 10^{-1}$ & - & $8,3 \cdot 10^{-3}$ & $6,6 \cdot 10^{-3}$ & $1,3 \cdot 10^{-2}$ \\
\hline & $\overline{\mathrm{x}}$ & $3,1 \cdot 10^{-2}$ & - & $6,1 \cdot 10^{-3}$ & $6,1 \cdot 10^{-3}$ & $1,3 \cdot 10^{-2}$ \\
\hline & $\sigma$ & $6,3 \cdot 10^{-2}$ & - & $2,2 \cdot 10^{-3}$ & $4,5 \cdot 10^{-4}$ & $3,1 \cdot 10^{-4}$ \\
\hline \multirow[t]{4}{*}{ 2-Ethylhexansäure } & Min & $1,1 \cdot 10^{-2}$ & $1,1 \cdot 10^{-1}$ & $4,2 \cdot 10^{-2}$ & - & $2,8 \cdot 10^{-2}$ \\
\hline & Max & $4,3 \cdot 10^{-2}$ & - & - & - & - \\
\hline & $\overline{\mathrm{x}}$ & $2,4 \cdot 10^{-2}$ & - & - & - & - \\
\hline & $\sigma$ & $1,2 \cdot 10^{-2}$ & - & - & - & - \\
\hline \multirow[t]{4}{*}{ Sebacinsäure ${ }^{b, l, n, p}$} & Min & $2,2 \cdot 10^{-2}$ & $6,7 \cdot 10^{-2}$ & - & - & - \\
\hline & Max & 5,4 & - & - & - & - \\
\hline & $\overline{\mathrm{x}}$ & 2,1 & - & - & - & - \\
\hline & $\sigma$ & 2,2 & - & - & - & - \\
\hline \multirow[t]{4}{*}{ Benzoesäure $e^{e, i, s, t, v}$} & Min & $4,9 \cdot 10^{-2}$ & $1,1 \cdot 10^{-2}$ & 2,7 & $1,1 \cdot 10^{-2}$ & $3,3 \cdot 10^{-2}$ \\
\hline & Max & 4,3 & $5,1 \cdot 10^{-2}$ & - & - & - \\
\hline & $\overline{\mathrm{x}}$ & $8,5 \cdot 10^{-1}$ & $3,1 \cdot 10^{-2}$ & - & - & - \\
\hline & $\sigma$ & 1,3 & $2,8 \cdot 10^{-2}$ & - & - & - \\
\hline
\end{tabular}

${ }^{\mathrm{a} C a r n e g i e ~ u n d ~ R a m s a y ~(2009) ; ~}{ }^{\mathrm{b}}$ Chapman und Duggleby (1967); ${ }^{\mathrm{c} C h u a ~ e t ~ a l . ~(2001) ; ~}{ }^{\mathrm{d}} \mathrm{EPA}$ (2016); ${ }^{\mathrm{e}}$ Gibson und Sulfita (1986); ${ }^{\mathrm{f}} \mathrm{Heesel}$ (2010); ${ }^{\mathrm{g}}$ Herzog et al. (2013); ${ }^{\mathrm{h}} \mathrm{llieva}$ et al. (2012); ${ }^{\mathrm{i}}$ Kazumi et al. (1995); ${ }^{\mathrm{j}}$ Liu et al. (2011); ${ }^{\mathrm{K}}$ Liu et al. (2013); ${ }^{\mathrm{l}}$ Matthies und Schink (1993); ${ }^{\mathrm{m}} \mathrm{McGahey}$ und

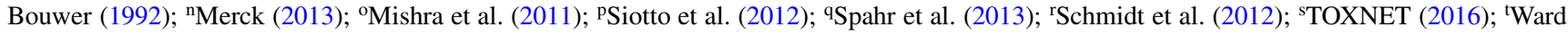
(1985); ${ }^{\text {u} W e i s s ~ e t ~ a l . ~(2006) ; ~}{ }^{\mathrm{v}}$ Williams und Evans (1975)

viel kleiner als die Elektronenakzeptorkonzentration ist und geringe Elektronenakzeptorkonzentrationen $(\geq 1 \mathrm{mg} / \mathrm{l})$ ausreichen, um einen maximalen stoffspezifischen Abbau zu erzielen.

$$
\begin{aligned}
\frac{d C_{\mathrm{Stoff}}}{d t} & =-k_{1 \mathrm{NO} 3} \cdot C_{\mathrm{Stoff}}\left(\frac{C_{\mathrm{NO} 3}}{K_{\mathrm{H}}+C_{\mathrm{NO} 3}}\right) \\
& \cong-k_{1 \mathrm{NO} 3} \cdot C_{\text {Stoff }} \text { für } K_{\mathrm{H}} \ll C_{\mathrm{NO} 3}
\end{aligned}
$$

Der Abbau durch Sulfatreduktion verläuft analog zum Abbau durch Nitratreduktion (Gl. 2). Als Elektronenakzeptorkonzentrationen wurden in Szenarien mit Nitratreduktion initial $10 \mathrm{mg} / \mathrm{l}$ Nitrat und in Szenarien mit Sulfatreduktion initial $50 \mathrm{mg} / \mathrm{l}$ Sulfat für den gesamten Grundwasserleiter gewählt.

Die Reaktion der Elektronenakzeptoren mit den Wärmeträgerfluid-Inhaltsstoffen wird im Modell über die Stöchiometrie der Abbaureaktion berücksichtigt, die am Beispiel von Ethylenglykol unter der Annahme des vollständigen
Abbaus zu $\mathrm{CO}_{2}$ für nitratreduzierende und sulfatreduzierende Bedingungen durch folgende Reaktionsgleichungen beschrieben wird:

$$
\begin{aligned}
\text { nitratreduzierend : } & \mathrm{C}_{2} \mathrm{H}_{4}(\mathrm{OH})_{2}+2 \mathrm{NO}_{3}^{-}+2 \mathrm{H}^{+} \\
& \rightarrow 2 \mathrm{CO}_{2}+4 \mathrm{H}_{2} \mathrm{O}+\mathrm{N}_{2} \\
\text { sulfatreduzierend : } & 4 \mathrm{C}_{2} \mathrm{H}_{4}(\mathrm{OH})_{2}+5 \mathrm{SO}_{4}^{2-}+10 \mathrm{H}^{+} \\
& \rightarrow 8 \mathrm{CO}_{2}+5 \mathrm{H}_{2} \mathrm{~S}+12 \mathrm{H}_{2} \mathrm{O}
\end{aligned}
$$

Um den Einfluss des durch die Trinkwasserentnahme entstandenen Grundwasserströmungsfeldes auf das Ausbreitungsverhalten der Stoffe zu untersuchen, wurden Abstand und Position der Erdwärmesonde zur Trinkwasserentnahme variiert und die maximalen Stoffkonzentrationen im Entnahmebrunnen anhand einer Durchbruchskurve ausgegeben. Die Erdwärmesonde wurde hierbei mit einem Abstand von $1000 \mathrm{~m}, 500 \mathrm{~m}$ oder $100 \mathrm{~m}$ von den Entnahmebrunnen direkt anstromig (EWS1), $45^{\circ}$ zur Achse der Entnahmebrunnen im Anstrom (EWS2), in Verlängerung 
Tab. 3 Im Modell angenommene Konzentrationen und Abbauratenkonstanten (Mittelwerte, siehe Tab. 2) der Inhaltsstoffe

Table 3 Concentrations assumed in the model and degradation rate constants (mean values, see table 2) of the constituents

Abb. 2 Skizze des Modellaufbaus. Grundwassergeringleiter wurden nicht mit modelliert

Fig. 2 Sketch of the model. Aquitards are not included

\begin{tabular}{lllll}
\hline & Konzentration $[\mathrm{mg} / \mathrm{l}]$ & $\mathrm{k}_{1 \mathrm{ferm}}\left[\mathrm{d}^{-1}\right]$ & $\mathrm{k}_{1 \mathrm{NO} 3}\left[\mathrm{~d}^{-1}\right]$ & $\mathrm{k}_{1 \mathrm{SO} 4}\left[\mathrm{~d}^{-1}\right]$ \\
\hline Ethylenglykol & $8 \cdot 10^{5 \mathrm{a}}$ & $3,6 \cdot 10^{-2}$ & $1,4 \cdot 10^{-1}$ & $1,6 \cdot 10^{-2}$ \\
Benzotriazol & $1,1 \cdot 10^{3 \mathrm{~b}}$ & - & $5,0 \cdot 10^{-3}$ & $3,8 \cdot 10^{-3}$ \\
Tolyltriazol & $3,5 \cdot 10^{3} \mathrm{a}$ & - & $6,1 \cdot 10^{-3}$ & $6,1 \cdot 10^{-3}$ \\
2-Ethylhexansäure & $1,75 \cdot 10^{4} \mathrm{a}$ & $1,1 \cdot 10^{-1}$ & $4,2 \cdot 10^{-2}$ & - \\
Sebacinsäure & $1,28 \cdot 10^{4} \mathrm{a}$ & $6,7 \cdot 10^{-2}$ & - & - \\
Benzoesäure & $9,5 \cdot 10^{3 \mathrm{a}}$ & $3,1 \cdot 10^{-2}$ & 2,7 & $1,1 \cdot 10^{-2}$ \\
\hline
\end{tabular}

allieva et al. (2014); b Schmidt et al. (2012)

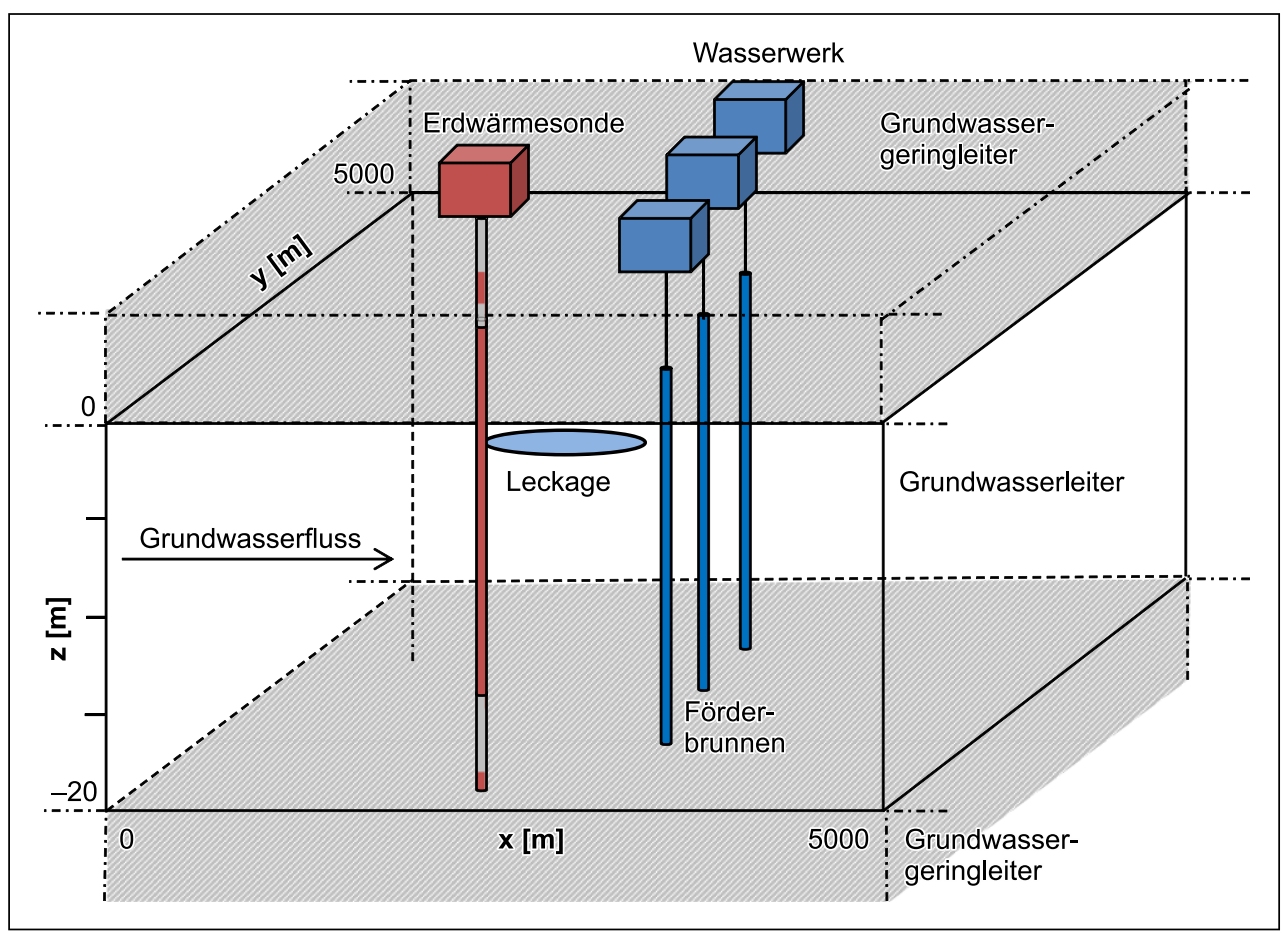

der Brunnenachse (EWS3) und abstromig (EWS4) positioniert (Abb. 3).

Weiterhin wurde in ergänzenden Szenarien die Gesamtentnahmerate der Trinkwassergewinnungsanlage von $1.000 .000 \mathrm{~m}^{3} / \mathrm{a}$ auf $500.000 \mathrm{~m}^{3} / \mathrm{a}, \quad 250.000 \mathrm{~m}^{3} / \mathrm{a}$ und $125.000 \mathrm{~m}^{3} / \mathrm{a}$ verringert, um den Einfluss unterschiedlicher Strömungs- und Verdünnungsverhältnisse auf die Stoffkonzentration zu analysieren.

Der Durchlässigkeitsbeiwert $\left(\mathrm{k}_{\mathrm{f}}\right)$ und die Porosität wurden jeweils im mittleren Bereich von LockergesteinsAquiferen aus Busch und Luckner (1974) zitiert nach Garling und Dittrich (1979) und Dethlefsen et al. (2017) variiert, um den Einfluss der hydraulischen Bedingungen auf die Stoffkonzentration zu untersuchen. Dafür wurden sowohl ein höherer $\mathrm{k}_{\mathrm{f}}$-Wert $\left(3 \cdot 10^{-3} \mathrm{~m} / \mathrm{s}\right)$ und eine größere Porosität $(25 \%)$, sowie auch ein geringerer $\mathrm{k}_{\mathrm{f}}-$ Wert $\left(3 \cdot 10^{-5} \mathrm{~m} / \mathrm{s}\right)$ und eine kleinere Porosität $(8 \%)$ als in den sonstigen Szenarien $\left(5 \cdot 10^{-4} \mathrm{~m} / \mathrm{s}, 20 \%\right)$ verwendet. Zudem wurde die Dispersivität aus Gelhar et al. (1985) zitiert nach Rausch et al. (2002) für eine Längen- skala von $100 \mathrm{~m}$ variiert, wobei sowohl 10 -fach höhere $\left(\alpha_{\mathrm{L}}=100 \mathrm{~m}, \alpha_{\mathrm{T}}=10 \mathrm{~m}\right)$ als auch 10-fach niedrigere Dispersivitäten $\left(\alpha_{\mathrm{L}}=1 \mathrm{~m}, \alpha_{\mathrm{T}}=0,1 \mathrm{~m}\right)$ betrachtet wurden.

Aus den Durchbruchskurven für die Entnahmebrunnen wurde die Maximalkonzentration der Stoffe extrahiert und mit den predicted no effect concentrations (PNEC) bzw. den Geringfügigkeitsschwellen (GFS) nach LAWA (Tab. 1) als Richtwert verglichen, da für die meisten Wärmeträgerfluid-Inhaltsstoffe keine Grenzwerte im Grundwasser nach der Trinkwasserverordnung (TrinkwV 2001) oder dem Bundes-Bodenschutzgesetz (BBodSchG 1998) vorliegen. Der PNEC-Wert ist die Konzentration unterhalb der höchstwahrscheinlich kein negativer Effekt für das Ökosystem auftritt. Dieser wird auf Grundlage der chronischen Toxizität (NOEC $=$ no effect concentration) für den empfindlichsten Organismus von drei Stufen der Nahrungskette, den so genannten trophischen Stufen (Pflanzen: Alge, Wirbellose: Daphnie, Wirbeltier: Fisch, Reduzenten: Bakterien) zuzüglich eines Sicherheitsfaktors (AF $=$ Assessment Factor) berechnet. Bei Betrachtung von drei trophischen Stufen liegt 


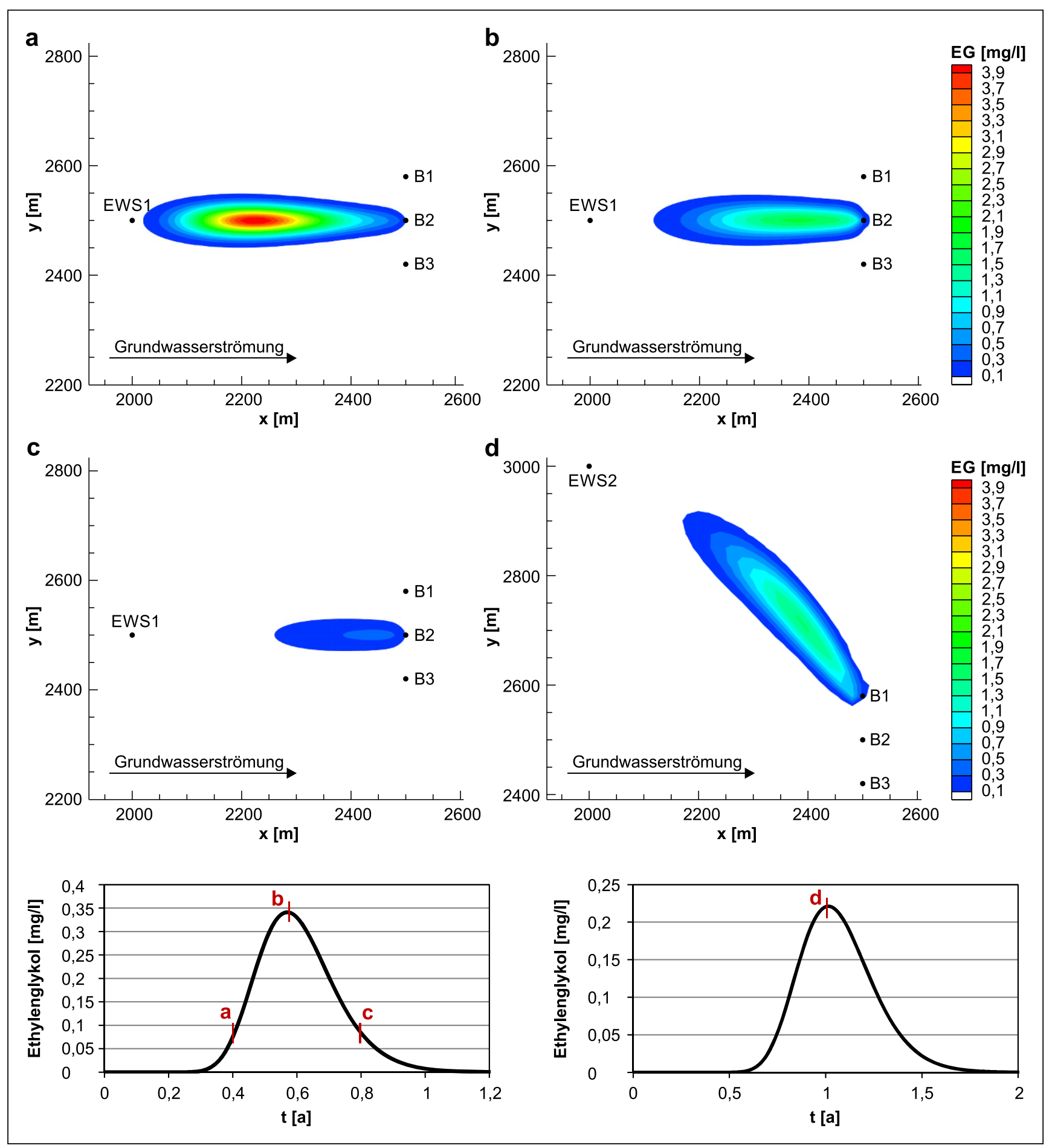

Abb. 3 Beispiel für Leckagen und Durchbruchskurven im Brunnen anhand des konservativen Transportes von Ethylenglykol (EG) bei einer Entnahmerate von $1.000 .000 \mathrm{~m}^{3} / \mathrm{a}$. Die Kartendarstellung zeigt den im Diagramm markierten Zeitpunkt

Fig. 3 Example of leakages and breakthrough curves in the well using the conservative transport of ethylene glycol (EG) at a withdrawal rate of $1,000,000 \mathrm{~m}^{3} / \mathrm{a}$. The map shows the time marked in the diagram 


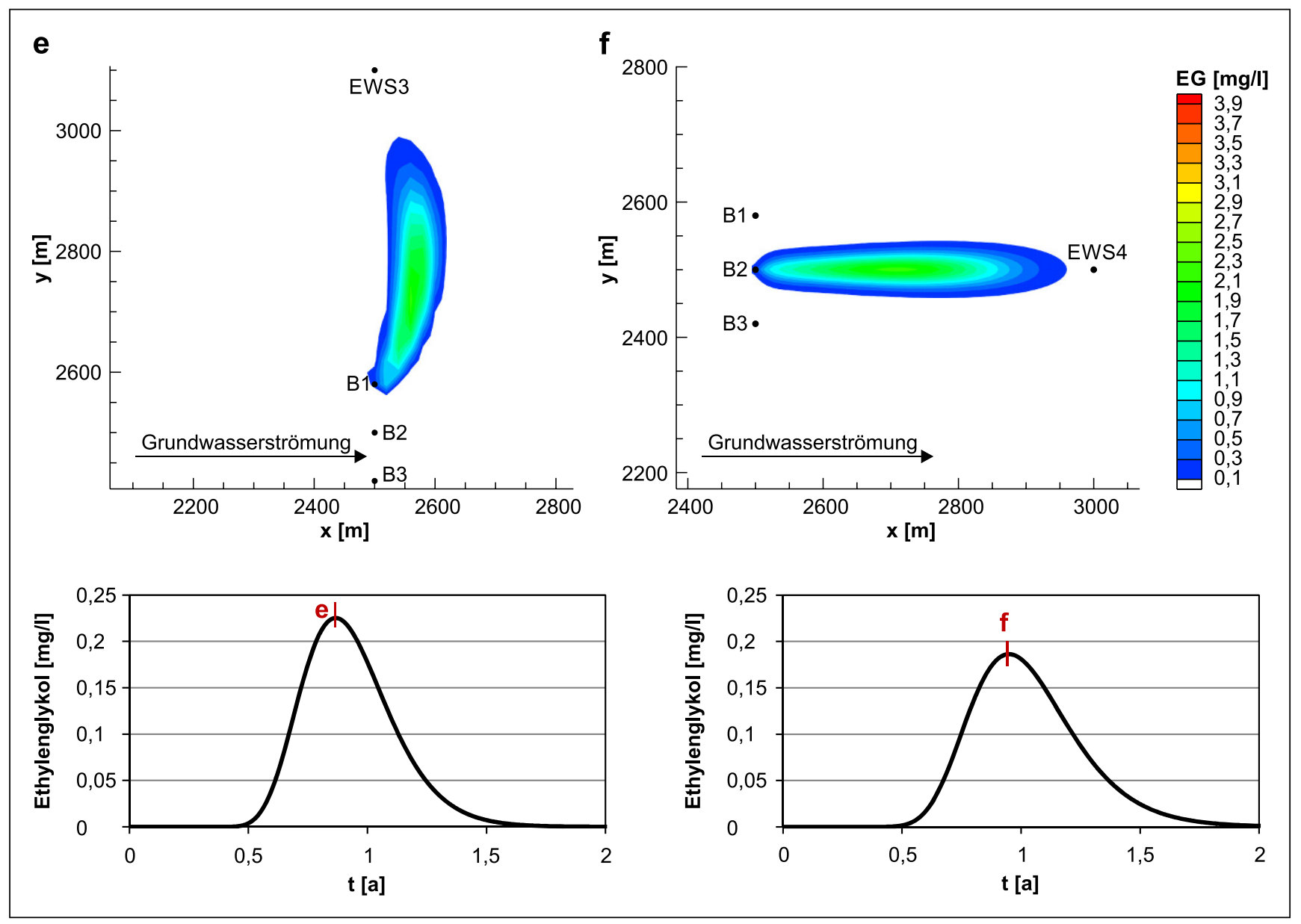

Abb. 3 (Fortsetzung)

Fig. 3 (Continued)

dieser Sicherheitsfaktor bei 10 und für zwei trophische Stufen bei 50 (LAWA 2004). Die Ableitung des GFS erfolgt auf Basis von human- und ökotoxikologischen Daten (Grenzwert der TrinkwV, Basisdaten Toxikologie, Umweltqualitätsnorm, PNEC) und leitet sich aus dem jeweils kleineren und daher meist dem ökotoxischen Wert ab, da dieser in der Regel bei niedrigeren Belastungen auftritt als der humantoxische. Somit entspricht der PNEC in den meisten Fällen dem GFS und kann daher als Richtwert herangezogen werden, wenn keine Geringfügigkeitsschwelle angegeben ist.

Da ca. $99 \%$ der Stofffahne jeweils nur in den nächstgelegenen der drei Brunnen fließt, wird im Folgenden jeweils nur der Brunnen mit der höchsten Konzentration an Wärmeträgerfluid-Inhaltsstoffen betrachtet.

\section{Ergebnisse}

Die Simulationen zeigen, dass bei einer Entnahmerate von $1.000 .000 \mathrm{~m}^{3} / \mathrm{a}$ bereits bei konservativem Transport und einem sehr geringen Abstand von nur $100 \mathrm{~m}$ zur Erdwärme- sonde (EWS1) aufgrund der hohen Verdünnung im Entnahmebrunnen (B2) die Konzentrationen, mit Ausnahme von Sebacinsäure, mit einem Faktor $\geq 10$ unterhalb der Richtwerte lagen (Abb. 4). Durch die zusätzliche Berücksichtigung von mikrobiellem Abbau ergaben sich entsprechend geringere Konzentrationen, sodass diese im Entnahmebrunnen in Abhängigkeit vom Redoxmilieu mit einem Faktor $\geq 14$, bei Benzoesäure sogar mit einem Faktor $>1000$ unterhalb des Richtwerts lagen (Abb. 4). Sebacinsäure unterschreitet beim konservativen Transport den Richtwert mit einem Faktor von 1,2 und unter Berücksichtigung von mikrobiellem Abbau mit einem Faktor 3, wobei der Abbau von Sebacinsäure aufgrund der begrenzten Datengrundlage nur mit höheren Unsicherheiten berücksichtigt werden konnte. Für Ethylenglykol erfolgte mit einer um $67 \%$ niedrigeren Konzentration als beim konservativen Transport der höchste Abbau durch Nitratreduktion, gefolgt vom Abbau durch Fermentation (-28\%) und Sulfatreduktion $(-23 \%)$. Es zeigte sich, dass die relative Konzentrationsverringerung durch Mitbetrachtung des Abbaus für das eigentlich gut abbaubare Ethylenglykol aufgrund der Limitierung durch Elektro- 
nenakzeptoren ( $<1 \mathrm{mg} / \mathrm{l}$ Nitrat im Zentrum der Stofffahne) geringer ist als für die Substanzen mit geringeren Abbauratenkonstanten. Für Benzotriazol ergab sich durch den Abbau durch Nitratreduktion eine um $89 \%$ und durch Sulfatreduktion eine um $81 \%$ niedrigere Konzentration als beim konservativen Transport. Eine ähnliche Konzentrationsverringerung durch anaeroben Abbau zeigte sich bei Tolyltriazol, wobei die Konzentration sowohl durch Nitrat- als auch Sulfatreduktion um $93 \%$ niedriger sind als bei konservativem Transport. 2-Ethylhexansäure weist im Vergleich zum konservativen Transport entsprechend der Abbauratenkonstanten durch Nitratreduktion $62 \%$ und durch fermentativen Abbau um 74\% niedrigere Konzentrationen auf. Bei Sebacinsäure lag die Konzentration durch Berücksichtigung von Fermentation um 53\% niedriger als bei konservativem Transport. Benzoesäure zeigte eine sehr gute Abbaubarkeit durch Nitratreduktion $\left(2,67 \mathrm{~d}^{-1}\right)$, weshalb hier im Vergleich zum konservativen Transport die Konzentration um 99,9\% niedriger lag. Durch fermentativen Abbau ergaben sich um $23 \%$ niedrigere und durch Sulfatreduktion gemäß der geringeren Abbauratenkonstanten um 12\% niedrigere Konzentrationen im Entnahmebrunnen.

\section{Variation der Entnahmerate}

Die Variation der Entnahmerate zur Untersuchung des Einflusses der Dispersion und Verdünnung zeigte am Beispiel von Ethylenglykol, dass durch eine Verringerung der Entnahmerate von $1.000 .000 \mathrm{~m}^{3} / \mathrm{a}$ auf $125.000 \mathrm{~m}^{3} / \mathrm{a}$ bei konservativem Transport bei allen betrachteten Abständen von der Erdwärmesonde zum Entnahmebrunnen (100 m, 500 m, $1000 \mathrm{~m})$ eine dreifach erhöhte Konzentration im Entnahmebrunnen auftrat (Abb. 5b). Bei einem Abstand von nur $100 \mathrm{~m}$ vom Entnahmebrunnen lag beispielsweise beim konservativen Transport von Ethylenglykol die Konzentration bei einer Entnahmerate von $500.000 \mathrm{~m}^{3} / \mathrm{a}$ mit einem Faktor von 6 unter dem Richtwert, bei einer Entnahme von $250.000 \mathrm{~m}^{3} / \mathrm{a}$ mit einem Faktor von 4 und bei einer Entnahme von $125.000 \mathrm{~m}^{3} / \mathrm{a}$ nur noch mit einem Faktor von 3 (Abb. 5a). Dennoch konnte auch bei einer geringen Entnahmerate von $125.000 \mathrm{~m}^{3} / \mathrm{a}$ und einem Abstand von nur $100 \mathrm{~m}$, bei allen Substanzen mit Ausnahme von Sebacinsäure, keine Überschreitung des Richtwertes festgestellt werden (Abb. 4). Die Konzentrationen lagen bei diesem Szenario mit konservativem Transport mit einem Faktor von $\geq 3$ unterhalb der Richtwerte. Sebacinsäure lag hingegen einzig bei diesem Szenario mit konservativem Transport mit einem Faktor von 3 über dem Richtwert. Durch die Berücksichtigung von mikrobiellem Abbau ergaben sich für alle betrachteten Substanzen bei der Entnahmerate von $125.000 \mathrm{~m}^{3} / \mathrm{a}$ geringere Konzentrationen als beim konservativen Transport, die vielfach mit einem Faktor $>1000$ unterhalb des Richtwerts lagen (Abb. 4, $b$ )). Bei Ethylenglykol zeigte sich, dass der Abbau durch Nitratreduktion aufgrund einer hohen Nitratzehrung $(<1 \mathrm{mg} / \mathrm{l}$ Nitrat im Zentrum der Stofffahne, Abb. 6) limitiert wurde und die Konzentrationen im Vergleich zum konservativen Transport dadurch nur um $40 \%$ niedriger waren. Der Abbau durch Fermentation ergab um $93 \%$ niedrigere und durch Sulfatreduktion um $92 \%$ niedrigere Konzentrationen als beim konservativen Transport. Bei Benzotriazol und Tolyltriazol ergab sich durch Nitrat- und Sulfatreduktion eine Abweichung der Konzentration vom konservativen Transport um -99,8 bis $-99,9 \%$. Auch bei 2-Ethylhexansäure ergab der Abbau durch Nitratreduktion um $96 \%$ und durch Fermentation um 99,9\% niedrigere Konzentrationen als beim konservativen Transport und ist somit mit einem Faktor von bis zu 162 effektiver als bei der höheren Pumprate $\left(1.000 .000 \mathrm{~m}^{3} / \mathrm{a}\right)$. Sebacinsäure zeigte eine zu 99,3\% niedrigere Konzentration durch fermentativen Abbau im Vergleich zum konservativen Transport, bei der höheren Pumprate lag diese nur bei einer Abweichung von $-53 \%$. Bei Benzoesäure ergab der Abbau durch Nitratreduktion eine um 99,99\% (> Faktor 1000) geringere Konzentration als beim konservativen Transport. Durch Fermentation ergab sich eine Abweichung der Konzentration im Vergleich zum konservativen Transport um $-89 \%$, durch Sulfatreduktion um $-75 \%$.

Es zeigte sich, dass aufgrund der geringeren Entnahmerate von $125.000 \mathrm{~m}^{3} / \mathrm{a}$ und der damit verbundenen längeren Aufenthaltszeit der Abbau der Stoffe effektiver stattfand, sofern dieser nicht durch den Verbrauch von Elektronenakzeptoren limitiert wird. Eine Limitierung durch Elektronenakzeptoren zeigte sich beim Abbau von Ethylenglykol durch Nitratreduktion aufgrund einer hohen Nitratzehrung ( $<1 \mathrm{mg} / \mathrm{l} \mathrm{Nitrat} \mathrm{im} \mathrm{Zentrum} \mathrm{der} \mathrm{Stofffahne,} \mathrm{Abb.} \mathrm{6)} \mathrm{bei}$ einer Pumprate von $125.000 \mathrm{~m}^{3} / \mathrm{a}$, wodurch die Konzentrationen im Entnahmebrunnen nur um $40 \%$ niedriger waren als beim konservativen Transport. Bei der höheren Pumprate $\left(1.000 .000 \mathrm{~m}^{3} / \mathrm{a}\right)$ reduzierte sich die Konzentration hingegen um $67 \%$. Eine Limitierung durch die Elektronenakzeptorkonzentration konnte nur bei Ethylenglykol festgestellt werden, was darauf zurückzuführen ist, dass Ethylenglykol als Frostschutzmittel im Vergleich zu den Additiven in höheren Endkonzentrationen verwendet wird. Unter Berücksichtigung von Abbauprozessen ergaben sich daher bei einer Entnahmerate von $125.000 \mathrm{~m}^{3} / \mathrm{a}$ meist niedrigere Konzentrationen im Entnahmebrunnen (um bis zu Faktor 200, bei Benzoesäure sogar mit einem Faktor $>1000$ ) als bei einer Entnahmerate von $1.000 .000 \mathrm{~m}^{3} / \mathrm{a}$, während bei einer Nichtberücksichtigung von Abbauprozessen und der Annahme eines konservativen Transports aufgrund geringerer Verdünnung im Brunnen bei $125.000 \mathrm{~m}^{3} / \mathrm{a}$ höhere Konzentrationen vorlagen. 


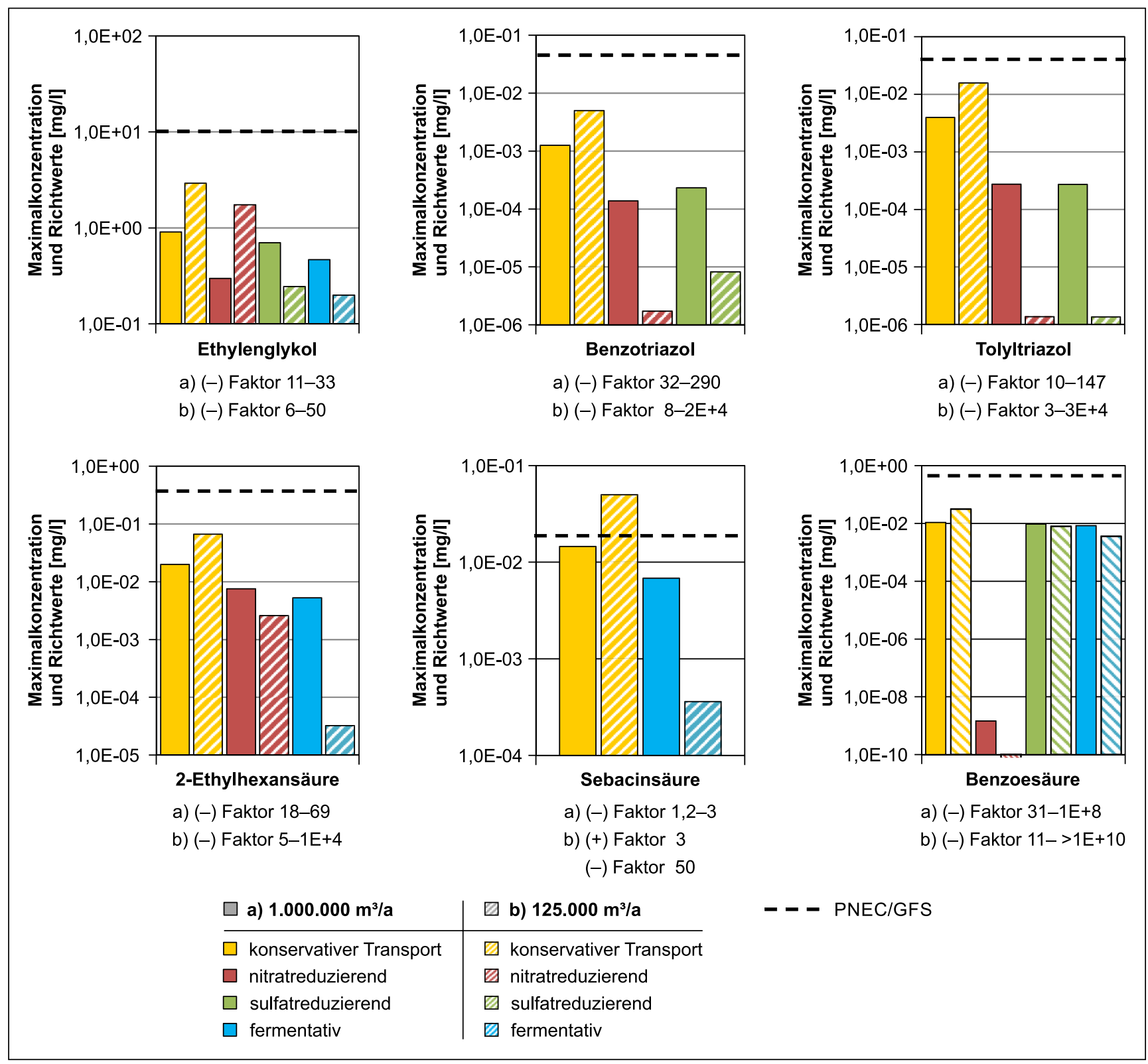

Abb. 4 Maximalkonzentration im Brunnen (B2) bei einem Abstand der Erdwärmesonde von $100 \mathrm{~m}$ im Anstrom (EWS1) im Vergleich zum Richtwert bei einer Entnahme von a) $1.000 .000 \mathrm{~m}^{3} / \mathrm{a}$ und b) $125.000 \mathrm{~m}^{3} / \mathrm{a}$. Der Faktor gibt an, wie weit die Maximalkonzentration den Richtwert unter- $(-)$ oder überschreitet $(+)$. Dabei ist der Faktor $=$ Richtwert/Maximalkonzentration

Fig. 4 Maximum concentration in the well (B2) at a borehole heat exchanger distance of $100 \mathrm{~m}$ in the inflow (EWS1) compared to the guide value for $a$ ) $1,000,000 \mathrm{~m}^{3} / \mathrm{a}$ and $\left.b\right) 125,000 \mathrm{~m}^{3} / \mathrm{a}$. The factor indicates how far the maximum concentration exceeds (+) or falls below (-) the guideline value. The factor $=$ guide value/maximum concentration

\section{Variation von Abstand und Position der Erdwärmesonde}

Bei der Variation von Abstand und Position der Erdwärmesonde wurde besonders bei höheren Abständen von 500 und $1000 \mathrm{~m}$ bis zur Trinkwasserentnahme deutlich, dass sich die Konzentrationen bei einer Positionierung im seitlichen und unterstromigen Bereich der Entnahme aufgrund des längeren Fließweges bzw. der längeren Fließzeit (siehe im Fol- genden) wesentlich verringern, da eine höhere Dispersion stattfindet (Abb. 5a). So lag beispielsweise beim konservativen Transport von Ethylenglykol bei einer Positionierung entlang der Brunnenachse (EWS3) die Konzentration im Entnahmebrunnen (B1) bei einem Abstand von $500 \mathrm{~m}$ mit einem Faktor von 45 (Konzentrationsmaximum im Brunnen nach 0,9 Jahren) und bei $1000 \mathrm{~m}$ Abstand und einer viermal längeren Fließzeit mit einem Faktor von 150 (Konzentrationsmaximum im Brunnen nach 3,6 Jahren) unter 
Abb. 5 Maximalkonzentration im Brunnen bei einem Abstand der Erdwärmesonde von 100, 500 und $1000 \mathrm{~m}$ im Anstrom im Vergleich zum Richtwert bei variierender Position der Erdwärmesonde (a) und Entnahmerate (b) am Beispiel von Ethylenglykol

Fig. 5 Maximum concentration in the well at a borehole heat exchanger distance of 100 , 500 and $1000 \mathrm{~m}$ in the inflow compared to the guide value at varying position of the borehole heat exchanger (a) and sampling rate (b) using the example of ethylene glycol

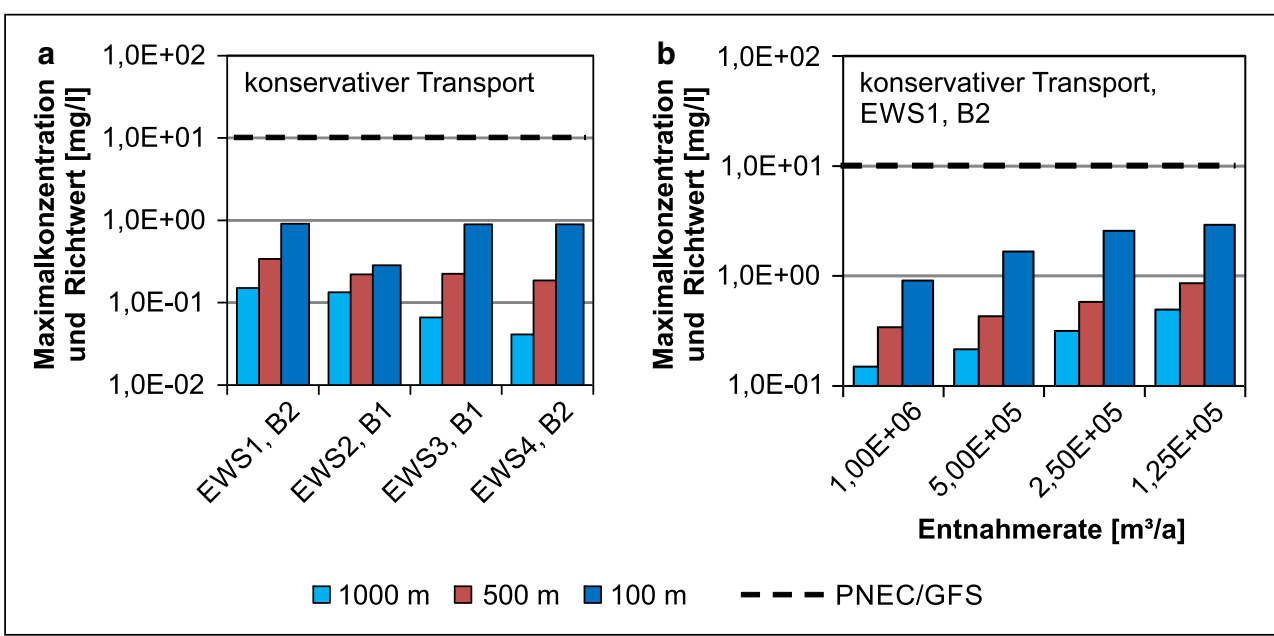

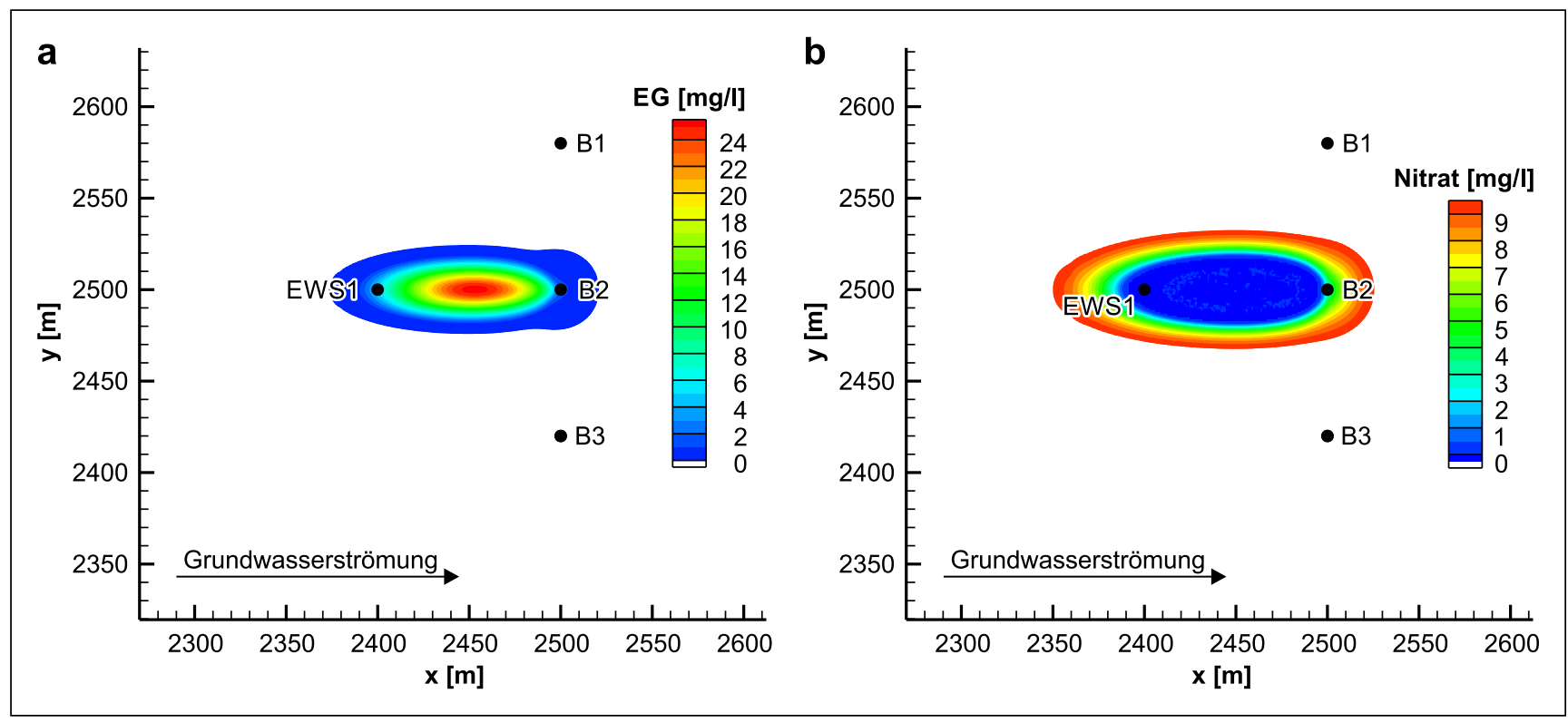

Abb. 6 a Ethylenglykol- (EG) und b Nitratverteilung bei Leckage von Ethylenglykol aus der Erdwärmesonde EWS1 unter nitratreduzierenden Bedingungen mit einer Nitratzehrung bei einer Entnahmerate von $125.000 \mathrm{~m}^{3} / \mathrm{a}$ und einem Abstand der Erdwärmesonde von $100 \mathrm{~m}$ im Anstrom des Brunnens nach 85 Tagen (Zeitpunkt der Maximalkonzentration von Ethylenglykol im Brunnen)

Fig. 6 a Distribution of ethylene glycol (EG) and b nitrate in case of ethylene glycol leakage from borehole heat exchanger EWS1 under nitrate-reducing conditions with a nitrate consumption at a withdrawal rate of $125,000 \mathrm{~m}^{3} / \mathrm{a}$ and a distance of the borehole heat exchanger of $100 \mathrm{~m}$ in the inflow of the well after 85 days (time of maximum concentration of ethylene glycol in the well)

dem Richtwert, während diese vergleichsweise bei einer direkten Positionierung der Sonde im zentralen Anstrom zur Entnahme (EWS1) bei einem Abstand von $500 \mathrm{~m}$ mit einem Faktor von 30 (Konzentrationsmaximum im Brunnen nach 0,9 Jahren) und bei einem Abstand von $1000 \mathrm{~m}$ und einer doppelt so langen Fließzeit mit einem Faktor von 70 (Konzentrationsmaximum im Brunnen nach 1,8 Jahren) unter dem Richtwert lagen (Abb. 5). Bei einer Positionierung im zentralen Abstrom (EWS4) lag die Konzentration im Entnahmebrunnen (B2) bei einem Abstand von $500 \mathrm{~m}$ sogar mit einem Faktor von 55 (Konzentrationsmaximum im Brunnen nach 1,0 Jahren) und bei $1000 \mathrm{~m}$ Abstand und einer fünf- mal längeren Fließzeit mit einem Faktor von 240 (Konzentrationsmaximum im Brunnen nach 5,3 Jahren) unter dem Richtwert. Bei einem geringeren Abstand von nur $100 \mathrm{~m}$ zeigte sich kaum noch ein Einfluss der Position der Erdwärmesonde auf die Konzentrationen im Entnahmebrunnen, sodass bei einer zentralen Positionierung im Anstrom der Entnahme (EWS1) (Konzentrationsmaximum im Brunnen nach 0,1 Jahren), als auch bei einer Positionierung entlang der verlängerten Brunnenachse (EWS3) (Konzentrationsmaximum im Brunnen nach 0,1 Jahren) oder zentral unterstromigen (EWS4) (Konzentrationsmaximum im Brunnen nach 0,1 Jahren) Positionierung die Konzentratio- 
nen immer noch in etwa mit einem Faktor von 10 unter dem Richtwert lagen. Nur bei einer Positionierung im $45^{\circ}$-Winkel zur Achse der Entnahmebrunnen im Anstrom (EWS2) ergibt sich eine längere Aufenthaltszeit und somit niedrigere Konzentrationen im Entnahmebrunnen (B1), die mit einem Faktor von 35 (Konzentrationsmaximum im Brunnen nach 0,2 Jahren) unter dem Richtwert liegen.

\section{Variation hydraulischer Parameter}

Eine Variation des $\mathrm{k}_{\mathrm{f}}$-Wertes im mittleren Bereich von Lockergesteinsaquiferen ergab beim konservativen Transport von Ethylenglykol bei einem Abstand der Erdwärmesonde zum Entnahmebrunnen von $100 \mathrm{~m}$ für einen höheren $\mathrm{k}_{\mathrm{f}}$-Wert von $3 \cdot 10^{-3} \mathrm{~m} / \mathrm{s}$ im Vergleich zum ursprünglich angenommenen $\mathrm{k}_{\mathrm{f}}$-Wert $\left(5 \cdot 10^{-4} \mathrm{~m} / \mathrm{s}\right)$ eine Abweichung der Maximalkonzentration im Entnahmebrunnen um 1,5\%, für einen niedrigeren $\mathrm{k}_{\mathrm{f}}$-Wert von $3 \cdot 10^{-5} \mathrm{~m} / \mathrm{s}$ eine Abweichung um $-0,7 \%$. Eine höhere Porosität von $25 \%$ als ursprünglich angenommen (20\%) erbrachte eine Abweichung der Maximalkonzentration um $-1,6 \%$, eine niedrigere Porosität von $8 \%$ eine Abweichung von $0,9 \%$. Somit haben diese hydraulischen Bedingungen im Nahbereich der Erdwärmesonde erwartungsgemäß nur wenig Auswirkung auf die Konzentration im Förderbrunnen. Bei einer hohen Dispersivität $\left(\alpha_{\mathrm{L}}=100 \mathrm{~m}, \alpha_{\mathrm{T}}=10 \mathrm{~m}\right)$ ergibt sich eine Abweichung zur ursprünglich berechneten Maximalkonzentration (für $\left.\alpha_{\mathrm{L}}=10 \mathrm{~m}, \alpha_{\mathrm{T}}=1 \mathrm{~m}\right)$ von $-47,2 \%$, wogegen eine niedrige Dispersivität $\left(\alpha_{\mathrm{L}}=1 \mathrm{~m}, \alpha_{\mathrm{T}}=0,1 \mathrm{~m}\right)$ zu einer Konzentrationsabweichung von $+74,1 \%$ führt. Insgesamt zeigt sich also, dass die Variation der Entnahmerate die anderen Faktoren überprägt und wie beschrieben ausschlaggebend für die Verdünnung im Brunnen und die zum Abbau erforderliche Aufenthaltszeit ist.

\section{Diskussion}

Die exemplarischen numerischen Simulationen des Ausbreitungsverhaltens von Wärmeträgerfluid-Inhaltsstoffen aus Erdwärmesonden-Leckagen ins Grundwasser zeigten bereits bei Vernachlässigung von Abbau- sowie Sorptionsprozessen und ausschließlicher Annahme eines konservativen Transportes, dass bei einem Abstand von nur $100 \mathrm{~m}$ zwischen Erdwärmesonde und Trinkwasserbrunnen für alle Szenarien die Maximalkonzentrationen fast aller betrachteten Wärmeträgerfluid-Inhaltsstoffe im Brunnen aufgrund des hohen Verdünnungseffektes mit einem Faktor $>10$ unterhalb der Richtwerte lagen. Unter Berücksichtigung von mikrobiellem Abbau ergeben sich noch geringere Konzentrationen im Brunnen, die in Abhängigkeit der Redoxbedingungen größtenteils mit einem Faktor $>30$ und bei Benzoesäure sogar mit einem Faktor $>1000$ unter dem je- weiligen Richtwert lagen. Diese niedrigen Konzentrationen treten bei Benzoesäure aufgrund der hohen Abbauratenkonstante $\left(2,67 \mathrm{~d}^{-1}\right)$ unter nitratreduzierenden Bedingungen auf. Auch bei den anderen Substanzen, außer bei 2-Ethylhexansäure, zeigte sich die typische Abhängigkeit des Abbaus vom Redoxmilieu, bei der die Abbauraten von nitratreduzierenden zu fermentativen und sulfatreduzierenden Bedingungen hin abnehmen. Bei 2-Ethylhexansäure liegt für den fermentativen Abbau eine höhere Abbaurate vor als für den nitratreduzierenden, wobei sich diese Angaben nur auf die Ergebnisse jeweils einer Studie stützen und ohne Vergleichswerte mit einer gewissen Unsicherheit behaftet sind. Gleiches gilt für den Abbau von Sebacinsäure unter fermentativen Bedingungen, die mit einem Faktor von 3 die geringste Unterschreitung des Richtwertes aufwies.

Auch eine Verringerung der Grundwasserentnahmerate von zuvor $1.000 .000 \mathrm{~m}^{3} / \mathrm{a}$ auf $125.000 \mathrm{~m}^{3} / \mathrm{a}$ führte mit Ausnahme eines angenommenen konservativen Transportes von Sebacinsäure zu keiner Überschreitung des Richtwertes. Bei geringeren Entnahmeraten treten aufgrund der ca. doppelt so langen Aufenthaltszeit der Stoffe im Grundwasser bei der Berücksichtigung von Abbauprozessen bis zu $99,5 \%$, bei Benzoesäure sogar bis zu 99,9\%, niedrigere Konzentrationen im Entnahmebrunnen auf als bei einer Entnahmerate von $1.000 .000 \mathrm{~m}^{3} / \mathrm{a}$, sofern der Abbau nicht wie bei Ethylenglykol durch den Verbrauch von Elektronenakzeptoren limitiert wird. Eine solche Abbaulimitierung entsteht z.B. im Szenario mit nitratreduzierendem Ethylenglykolabbau bei einer geringeren Entnahmerate von $125.000 \mathrm{~m}^{3} / \mathrm{a}$ und der damit verbundenen längeren Aufenthaltszeit, da durch den hohen Ethylenglykoleintrag im Zentrum der Leckage die Nitratkonzentrationen auf $<1 \mathrm{mg} / 1$ sinken (Abb. 6). In einem solchen Fall würde jedoch der Abbau bei zu geringen Nitratkonzentrationen fermentativ erfolgen und sich dadurch die Ethylenglykol-Konzentrationen weiter verringern, sodass die Maximalkonzentrationen im Brunnen höchstens die Werte des Szenarios mit ausschließlich fermentativ angenommenem Abbau erreichen (vgl. Abb. 4).

Bei einer Nichtberücksichtigung reaktiver Prozesse und Annahme eines konservativen Transports traten aufgrund geringerer Verdünnung im Brunnen bei $125.000 \mathrm{~m}^{3} / \mathrm{a}$ hingegen um bis zu viermal höhere Konzentrationen auf als bei einer Entnahmerate von $1.000 .000 \mathrm{~m}^{3} / \mathrm{a}$. Unter aeroben Bedingungen, die hier nicht berücksichtigt wurden, sind aufgrund höherer Abbauratenkonstanten (siehe Abb. 2) sogar noch geringere Maximalkonzentrationen zu erwarten. Insgesamt zeigte sich, dass die Variation der Entnahmerate die anderen Faktoren überprägt und wie beschrieben ausschlaggebend für die Verdünnung und die zum Abbau erforderliche Aufenthaltszeit ist. Die Ergebnisse sind für Porengrundwasserleiter in den untersuchten $\mathrm{k}_{\mathrm{f}}$-Bereichen gültig und nicht auf Kluft- und Karstgrundwasserleiter übertrag- 
bar. Ein prinzipielles Modell ersetzt zudem nicht eine spezifische Standortuntersuchung.

Angesichts des großen Beitrags, den Erdwärmeanlagen und/oder saisonale unterirdische Wärmespeicher zur Emissionsminderung im noch immer weitgehend durch fossile Energieträger dominierten Wärmesektor liefern könnten, entstehen durch pauschale „Sicherheitsabstände“, bei denen 99\% der geschützten Fläche in den meisten Fällen nicht durch Erdwärmesonden-Leckagen gefährdet wären (ringförmige, durch 100-m- und 1000-m-Radius begrenzte Fläche), potenziell erhebliche Einschränkungen der Erdwärmesonden-Nutzung und des damit verbundenen Potenzials im urbanen Raum. Hilfreich wären daher länderübergreifende einheitliche Regelungen mit naturwissenschaftlich begründeten Abstandsvorgaben.

Nach den Ergebnissen der numerischen Simulationen ist die Beeinträchtigung der Trinkwasserversorgung durch kontaminiertes Grundwasser aufgrund einer Erdwärmesondenleckage auch bei wesentlich geringeren restriktiven „Sicherheitsabständen" ohne weitere Gegenmaßnahmen wenig wahrscheinlich. Aufgrund der hier gemachten Annahme eines kompletten Austritts des Wärmeträgerfluids aus der Erdwärmesonde, sind die Ergebnisse zudem eher konservativ zu bewerten. Ein kompletter Austritt des Wärmeträgerfluides in das Grundwasser sollte durch einen eingebauten Niederdruckpressostat in der Erdwärmesondenanlage, der den Druckabfall registriert und die Anlage daraufhin abschaltet, in der Regel verhindert werden. Selbst für den Fall eines doppelten Störfalls (Leckage aus der Erwärmesonde und Versagen des Sicherheitssystems), der gleichzeitig an mehreren Erdwärmesonden erscheint, sind, ohne es derzeit im Detail untersucht zu haben, eine Reihe von einfachen Interventionsverfahren möglich, um eine nachhaltige Schädigung des Grundwassers zu verhindern. $\mathrm{Zu}$ denken wäre u. a. an das einfache Verwerfen oder das Verschneiden der im Volumen begrenzten Wassermengen, da selbst für das angenommene unwahrscheinlich doppelte Störfallszenario i.d.R. jeweils nur ein Förderbrunnen kurzzeitig betroffen wäre. Obwohl nach den Ergebnissen dieser Arbeit auch die Menge und Art der eingesetzten Mittel zur Korrosionsverhinderung bereits heute nur ein relativ geringes Gefährdungspotenzial für das Grundwasser aufweisen, erscheint es aus Akzeptanzgründen erstrebenswert und möglich, im Rahmen von Technologieentwicklungen die Verwendung bzw. die potenziellen Umweltauswirkungen der Zuschlagstoffe zur Korrosionsverhinderung weiter zu reduzieren.

Generell besteht nicht zuletzt vor dem Hintergrund der Ergebnisse dieser Arbeit zudem Bedarf an einer generellen nationalen Diskussion, wie pauschale und naturwissenschaftlich nicht immer begründete Abstandsvorgaben zwischen Trinkwasserbrunnen und Erdwärmeanlagen und/oder saisonalen unterirdischen Wärmespeichern angesichts der dringenden Notwendigkeit von Klimaschutzmaßnahmen im
Wärmebereich miteinander vereinbart werden können. Methodisch existieren heute schon hinreichende numerische Simulationswerkzeuge, um entsprechende Risiken grundsätzlich und ortsspezifisch auch für „Worst Case“-Szenarien quantifizieren zu können und geeignete Monitoring- und gegebenenfalls auch Interventionskonzepte zu entwerfen. Aus Sicht der Autoren ist es daher eine wesentliche zukünftige Aufgabe hydrogeologischer Forschung in Deutschland, Konzepte zur Vereinbarkeit von Klima- und Grundwasserschutz auf naturwissenschaftlich-technischer Basis zu entwickeln und umzusetzen.

Funding Open Access funding provided by Projekt DEAL.

Open Access Dieser Artikel wird unter der Creative Commons Namensnennung 4.0 International Lizenz veröffentlicht, welche die Nutzung, Vervielfältigung, Bearbeitung, Verbreitung und Wiedergabe in jeglichem Medium und Format erlaubt, sofern Sie den/die ursprünglichen Autor(en) und die Quelle ordnungsgemäß nennen, einen Link zur Creative Commons Lizenz beifügen und angeben, ob Änderungen vorgenommen wurden.

Die in diesem Artikel enthaltenen Bilder und sonstiges Drittmaterial unterliegen ebenfalls der genannten Creative Commons Lizenz, sofern sich aus der Abbildungslegende nichts anderes ergibt. Sofern das betreffende Material nicht unter der genannten Creative Commons Lizenz steht und die betreffende Handlung nicht nach gesetzlichen Vorschriften erlaubt ist, ist für die oben aufgeführten Weiterverwendungen des Materials die Einwilligung des jeweiligen Rechteinhabers einzuholen.

Weitere Details zur Lizenz entnehmen Sie bitte der Lizenzinformation auf http://creativecommons.org/licenses/by/4.0/deed.de.

\section{Literatur}

\section{Verwendete Literatur}

Ad-Hoc-Arbeitsgruppe Geologie: Fachbericht zu bisher bekannten Auswirkungen geothermischer Vorhaben in den Bundesländern (2011)

BbodSchG: Bundes-Bodenschutzgesetz vom 17. März 1998 (BGBl. I S. 502), das zuletzt durch Artikel 3 Absatz 3 der Verordnung vom 27. September 2017 (BGB1. I S. 3465) geändert worden ist (1998)

BMWi: Entwicklung der erneuerbaren Energien in Deutschland im Jahr 2018 (AGEE-Stat) (2019)

Bonte, M., Stuyfzand, P.J., Hulsmann, A., Van Beelen, P.: Underground thermal energy storage: environmental risks and policy developments in the Netherlands and European Union. Ecol. Soc. 16(1), 22 (2011)

Busch, K.F., Luckner, L.: Geohydraulik. Enke, Stuttgart (1974)

Butscher, C., Huggenberger, P., Auckenthaler, A., Bänninger, D.: Risikoorientierte Bewilligung von Erdwärmesonden. Grundwasser 16(1), 13-24 (2011)

Carnegie, D., Ramsay, J.A.: Anaerobic ethylene glycol degradation by microorganisms in poplar and willow rhizospheres. Biodegradation 20(4), 551-558 (2009)

CG Chemikalien: Sicherheitsdatenblatt Tolyltriazol. Sicherheitsdatenblatt gemäß Verordnung (EG) Nr. 1907/2006 (REACH) (2016)

Chapman, P.J., Duggleby, R.G.: Dicarboxylic acid catabolism by bacteria. Biochem. J. 103, 7contd-9c (1967)

Chua, H., Yu, P.H.F., Lo, W., Sin, S.N.: The degradation of xenobiotic branched carboxylic acids in anaerobic sediment of the Pearl 
River in Southern China. Sci. Total. Environ. 266(1-3), 221-228 (2001)

Dethlefsen, F., Duttmann, R., Feeser, V., Nolde, M., Schwanebeck, M., Pfeiffer, W.T., Wang, B., Dahmke, A.: Geo-Datenlage und technische Randbedingungen im Konzext der Energie- und Massenspeicherung. In: Dethlefsen, F., Dahmke, A., Bauer, S. (Hrsg.) Konzepte und Methoden für eine quantitative Raumplanung des Untergrundes. Unveröffentlichter Entwurf eines Kompendiums als Anlage zum Endbericht des Verbundvorhabens ANGUS (2017)

DVGW: Deutscher Verein des Gas- und Wasserfachs e.V.: Positionspapier zur Erdwärmenutzung in Trinkwassereinzugsgebieten (2010)

ECHA: European Chemicals Agency: (2016). https://echa.europa.eu/ de/information-on-chemicals, Zugegriffen: 14. Nov. 2016

EPA: Environmental Protection Agency: HPVIS: high production volume chemicals information system., USA (2016). http://www. epa.gov/hpvis/, Zugegriffen: 14. Nov. 2016

FVEE: Forschungsverband Erneuerbare Energien: Erneuerbare Energien im Wärmesektor - Aufgaben, Empfehlungen und Perspektiven (2015)

Garling, F., Dittrich, J.: Gesteinsbemusterung. Deutscher Verlag für Grundstoffindustrie, Leipzig (1979)

Gelhar, L.W., Mantaglou, A., Welty, C., Rehfeldt, K.R.: Review of a field-scale physical solute transport process in saturated and unsaturated porous media. Research project 2485-5, EPRI EA-4190. Electric Power Research Institute, Palo Alto (1985)

GESTIS-Stoffdatenbank: Das Gefahrstoffinformationssystem der Deutschen Gesetzlichen Unfallversicherung (2016). http://gestis. itrust.de, Zugegriffen: 14. Nov. 2016

Gibson, S.A., Sulfita, J.M.: Extrapolation of biodegradation results to ground water aquifers: reductive dehalogenation of aromatic compounds. Appl. Environ. Microbiol. 52, 681-682 (1986)

Gooden, W.J.: Biodegradation of propylene glycol-based MIL-A8243D aircraft deicer. Final report: June 1995-June 1997. Department of chemical and bioresource engineering Colorado State University, Fort Collins, Colorado (1998)

Grimm, M., Stober, I., Kohl, T., Blum, P.: Damage event analysis of drilling borehole heat exchangers in Baden-Württemberg, Germany. Grundwasser 19(4), 275-286 (2014)

Heesel, V.: Biodegradation of benzotriazoles. Master thesis. Eberhardt Karls Universität, Tübingen (2010)

Hem, L.J., Weideborg, M., Schram, S.: Degradation and toxicity of additives to aircraft de-icing fluids; the effect of discharge of such fluids to municipal wastewater treatment plants. Proc. Water Environ. Fed. 2000(5), 419-433 (2000)

Herzog, B., Huber, B., Lemmer, H., Horn, H., Müller, E.: Analysis and in situ characterization of activated sludge communities capable of benzotriazole biodegradation. Environ Sci. Eur. 25(1), 31 (2013)

Hollingsworth, J., Sierra-Alvarez, R., Zhou, M., Ogden, K.L., Field, J.A.: Anaerobic biodegradability and methanogenic toxicity of key constituents in copper chemical mechanical planarization effluents of the semiconductor industry. Chemosphere 59(9), 1219-1228 (2005)

Horn, O., Nalli, S., Cooper, D., Nicell, J.: Plasticizer metabolites in the environment. Water Res. 38(17), 3693-3698 (2004)

Ilieva, D., Haderlein, S.B., Morasch, B: Risikominimierung beim Einsatz von Additiven in Wärmeträgerflüssigkeiten. Abschlussbericht (2012)

Ilieva, D., Haderlein, S.B., Morasch, B.: Grundwassergefährdungspotenzial von Additiven in Wärmeträgerflüssigkeiten aus Erdwärmesonden. Grundwasser 19(4), 263-274 (2014). https://doi.org/ 10.1007/s00767-014-0272-6

Jia, Y., Bakken, L.R., Breedveld, G.D., Aagaard, P., Frostegård, Å.: Organic compounds that reach subsoil may threaten groundwater quality; effect of benzotriazole on degradation kinetics and microbial community composition. Soil Biol. Biochem. 38(9), 2543-2556 (2006)
Kazumi, J., Haggblom, M.M., Young, L.Y.: Degradation of monochlorinated and nonchlorinated aromatic compounds under iron-reducing conditions. Appl. Environ. Microbiol. 61(11), 4069-4073 (1995)

Klecka, G.M., Carpenter, C.L., Landenberger, B.D.: Biodegradation of aircraft deicing fluids in soil at low temperatures. Ecotoxicol. Environ. Saf. 25(3), 280-295 (1993)

Klotzbücher, T., Kappler, A., Straub, K.L., Haderlein, S.B.: Biodegradability and groundwater pollutant potential of organic anti-freeze liquids used in borehole heat exchangers. Geothermics 36(4), 348-361 (2007)

Köber, R., Dörr, C., Lüders, K., Schäfer, D., Dahmke, A.: Geochemische Beeinflussungen des Grundwassers durch Wärmespeicherung. Geotherm. Energ. 82(2), 20-21 (2015)

Koenigsdorff, R., Reuß, M.: Erdwärmesonden als geothermische Wärmeübertrager. Technologie und Planungswerkzeuge. Statusseminar: Thermische Energiespeicherung - mehr Energieeffizienz zum Heizen und Kühlen, Freiburg, 2.-3. November 2006. (2006)

Kolditz, O., Bauer, S.: A process-oriented approach to computing multi-field problems in porous media. J. Hydroinformatics 6(3), 225-244 (2004)

Kolditz, O., Bauer, S., Bilke, L., Böttcher, N., Delfs, J.O., Fischer, T., Görke, U.J., Kalbacher, T., Kosakowski, G., McDermott, C.I., Park, C.H., Radu, F., Rink, K., Shao, H., Shao, H.B., Sun, F., Sun, Y.Y., Singh, A.K., Taron, J., Walther, M., Wang, W., Watanabe, N., Wu, Y., Xie, M., Xu, W., Zehner, B.: OpenGeoSys: An open-source initiative for numerical simulation of thermo-hydromechanical/chemical (THM/C) processes in porous media. Environ Earth Sci 67(2), 589-599 (2012)

Kunz, P.: Umwelt-Bioverfahrenstechnik. Springer, Berlin (2013)

LANU: Hydrogeologischer Bericht des Landesamtes für Natur und Umwelt Schleswig-Holstein Abteilung Geologie/Boden zur Bemessung eines Wasserschutzgebietes für das Wasserwerk Uetersen (2000)

LAWA: Bund/Länder-Arbeitsgemeinschaft Wasser, Thüringer Ministerium für Umwelt, Energie und Naturschutz: Ableitung von Geringfügigkeitsschwellenwerten für das Grundwasser (2004)

LAWA: Bund/Länder-Arbeitsgemeinschaft Wasser, Thüringer Ministerium für Umwelt, Energie und Naturschutz: Ableitung von Geringfügigkeitsschwellenwerten für das Grundwasser. NSOHeterozyklen (2010)

LAWA: Bund/Länder-Arbeitsgemeinschaft Wasser, Thüringer Ministerium für Umwelt, Energie und Naturschutz: Empfehlungen der LAWA für wasserwirtschaftliche Anforderungen an Erdwärmesonden und Erdwärmekollektoren (2011)

Liu, Y.S., Ying, G.G., Shareef, A., Kookana, R.S.: Biodegradation of three selected benzotriazoles under aerobic and anaerobic conditions. Water Res. 45(16), 5005-5014 (2011)

Liu, Y.S., Ying, G.G., Shareef, A., Kookana, R.S.: Biodegradation of three selected benzotriazoles in aquifer materials under aerobic and anaerobic conditions. J. Contam. Hydrol. 151, 131-139 (2013)

LLUR: Ministerium für Energiewende, Landwirtschaft, Umwelt und ländliche Räume des Landes Schleswig-Holstein: Gesamtplan Grundwasserschutz in Schleswig-Holstein (1998)

Lüders, K., Firmbach, L., Ebert, M., Dahmke, A., Dietrich, P., Köber, R.: Gas-phase formation during thermal energy storage in near-surface aquifers: experimental and modelling results. Environ Earth Sci 75(21), 1404 (2016)

Lüders, K., Dahmke, A., Fiedler, M., Köber, R.: Temperature influence on mobilisation and (re)fixation of trace elements and heavy metals in column tests with aquifer sediments from 10 to $70^{\circ} \mathrm{C}$. Water Res. 169, 115-266 (2020). https://doi.org/10.1016/j.watres. 2019.115266

Matthies, C., Schink, B.: Anaerobic degradation of long-chain dicarboxylic acids by methanogenic enrichment cultures. FEMS Microbiol. Lett. 111(2-3), 177-182 (1993) 
McGahey, C., Bouwer, E.J.: Biodegradation of ethylene glycol in simulated subsurface environments. Water Sci. Technol. 26(1-2), 41-49 (1992)

Merck: Sicherheitsdatenblatt Sebacinsäure. Sicherheitsdatenblatt gemäß Verordnung (EG). Nr. 1907/2006 (2013)

Mishra, S.: Aerobic biodegradation of Benzotriazoles and analysis of metabolites. Masterthesis. Universität Tübingen, Tübingen (2011)

OXEA: Sicherheitsdatenblatt 2-Ethylhexansäure (2015)

PhysProb: Physical properties database (2016). http://www.syrres. com. Zugegriffen: 14. Nov. 2016

Pillard, D.A., Cornell, J.S., Dufresne, D.L., Hernandez, M.T.: Toxicity of benzotriazole and benzotriazole derivates to three aquatic species. Water Res. 35(2), 557-560 (2001)

Rausch, R., Schäfer, W., Wagner, C.: Einführung in die Transportmodellierung im Grundwasser. Gebrüder Borntraeger, Stuttgart (2002)

Rollinson, G., Callely, A.G.: No evidence for the biodegradation of benzotriazole by elective culture or continuous enrichment. Biotechnol. Lett. 8(4), 303-304 (1986)

Roth: Sicherheitsdatenblatt Benzoesäure. Sicherheitsdatenblatt gemäß Verordnung (EG) Nr. 1907/2006 (2015a)

Roth: Sicherheitsdatenblatt Ethylenglykol Sicherheitsdatenblatt gemäß Verordnung (EG) Nr. 1907/2006 (2015b)

Rumohr, S.: Nutzung der oberflächennahen Geothermie in Hessen Zahlen und Kenngrößen. bbr 3, 46-52 (2009)

Saner, D., Juraske, R., Kübert, M., Blum, P., Hellweg, S., Bayer, P.: Is it only $\mathrm{CO}_{2}$ that matters? A life cycle perspective on shallow geothermal systems. Renew. Sustain. Energy Rev. 14(7), 1798-1813 (2010)

Schmidt, K.R., Augenstein, T., Betting, D., Tiehm, A.: Wärmeträgerfluide in der Geothermie. Exemplarische Gefährdungsabschätzung anhand von Strukturaufklärung, Abbaubarkeit und Toxizität. In: TZW: DVGW-Technologiezentrum Wasser (Hrsg.) Aktuelle Themen im Grundwasserschutz, Bd. 52, S. 129-185. (2012)

Schoenberg, T., Veltman, S., Switzenbaum, M.: Kinetics of anaerobic degradation of glycol-based Type I aircraft deicing fluids. Biodegradation 12, 59-68 (2001)

Siotto, M., Sezenna, E., Saponaro, S., Innocenti, F.D., Tosin, M., Bonomo, L., Mezzanotte, V.: Kinetics of monomer biodegradation in soil. J. Environ. Manag. 93(1), 31-37 (2012)

Spahr, S., Huntscha, S., Bolotin, J., Maier, M.P., Elsner, M., Hollender, J., Hofstetter, T.B.: Compound-specific isotope analysis of benzotriazole and its derivatives. Anal Bioanal Chem 405(9), 2843-2856 (2013)

TOXNET: Toxicology data network (2016). https://toxnet.nlm.nih.gov, Zugegriffen: 14. Nov. 2016

TrinkwV: Trinkwasserverordnung in der Fassung der Bekanntmachung vom 10. März 2016 (BGBl. I S. 459), die zuletzt durch Artikel 1 der Verordnung vom 3. Januar 2018 (BGB1. I S. 99) geändert worden ist (2001)

VDI-Richtlinie 4640: Thermische Nutzung des Untergrunds (2010)

Ward, T.E.: Characterizing the aerobic and anaerobic microbial activities in surface and subsurface soils. Environ. Toxicol. Chem. 4(6), 727-737 (1985)
Weiss, S., Jakobs, J., Reemtsma, T.: Discharge of three benzotriazole corrosion inhibitors with municipal wastewater and improvements by membrane bioreactor treatment and ozonation. Environ. Sci. Technol. 40, 7193-7199 (2006)

Williams, R.J., Evans, W.C.: The metabolism of benzoate by moraxella species through anaerobic nitrate respiration. Biochem. J. 148, $1-10(1975)$

\section{Weiterführende Literatur}

Blume, H.-P., Brümmer, G.W., Horn, R., Kandeler, E., Kögel-Knabner, I., Kretzschmar, R.: Scheffer/Schachtschabel. Lehrbuch der Bodenkunde. Springer, Berlin, Heidelberg (2010)

BMUB: Bundesministerium für Umwelt, Naturschutz, Bau und Reaktorsicherheit: Klimaschutzplan 2050: Klimaschutzpolitische Grundsätze und Ziele der Bundesregierung (2016)

Bretti, C., Crea, F., Foti, C., Sammartano, S.: Solubility and activity coefficients of acidic and basic nonelectrolytes in aqueous salt solutions. 2. solubility and activity coefficients of suberic, azelaic, and sebacic acids in $\mathrm{NaCl}(\mathrm{aq}),\left(\mathrm{CH}_{3}\right)_{4} \mathrm{NCl}(\mathrm{aq})$, and $\left(\mathrm{C}_{2} \mathrm{H}_{5}\right)_{4} \mathrm{NI}(\mathrm{aq})$ at different ionic strengths and at $\mathrm{t}=25^{\circ} \mathrm{C}$. J. Chem. Eng. Data 51, 1660-1667 (2006)

Brielmann, H., Lueders, T., Schreglmann, K., Ferraro, F., Avramov, M., Hammerl, V., Blum, P., Bayer, P., Griebler, C.: Shallow geothermal energy usage and its potential impacts on groundwater ecosystems. Grundwasser 16(2), 77-91 (2011)

BWP Bundesverband Wärmepumpe e.V.: Trotz Ölpreisverfall: Wärmepumpenabsatz 2015 stabil. Pressemitteilung vom 25. Januar $2016(2016)$

Gruden, C.L., Dow, S.M., Hernandez, M.T.: Fate and toxicity of aircraft deicing fluid additives through anaerobic digestion. Water Environ. Res. 73(1), 72-79 (2001)

Hähnlein, S., Blum, P., Bayer, P.: Oberflächennahe Geothermie - aktuelle rechtliche Situation in Deutschland. Grundwasser 16(2), 69-75 (2011)

Huggenberger, P., Epting, J., Affolter, A., Zechner, E.: Concepts for transboundary groundwater management in a region of extensive groundwater use and numerous contaminated sites. International Conference "Transboundary Aquifers: Challenges and New Directions" (ISARM2010), Paris, 6.-8.12.2010. (2010)

Schmidt, K.R., Tiehm, A., Körner, B., Sacher, F., Conrad, R., Hollert, H.: Biodegradability and ecotoxicity of commercially available geothermal heat transfer fluids. Grundwasser 21(1), 59-67 (2016)

Stober, I., Bucher, K.: Geothermie. Springer Spektrum, Berlin (2014)

Umweltbundesamt: Erneuerbare Energien in Deutschland - Daten zur Entwicklung im Jahr 2017. Umweltbundesamt, Dessau-Roßlau (2018)

Hinweis des Verlags Der Verlag bleibt in Hinblick auf geografische Zuordnungen und Gebietsbezeichnungen in veröffentlichten Karten und Institutsadressen neutral. 